TRANSACTIONS OF THE

AMERICAN MATHEMATICAL SOCIETY

Volume 360, Number 9, September 2008, Pages 4497-4517

S 0002-9947(08)04552-2

Article electronically published on April 4, 2008

\title{
THE TWISTOR SECTIONS ON THE WOLF SPACES
}

\author{
YASUYUKI NAGATOMO
}

\begin{abstract}
Let $M$ be a compact quaternion symmetric space (a Wolf space) and $V \rightarrow M$ an irreducible homogeneous vector bundle on $M$ with its canonical connection, whose rank is less than or equal to the dimension of $M$. We classify the zero loci of the transversal twistor sections with a reality condition. There exists a bijection between such zero loci and the real representations of simple compact connected Lie groups with non-trivial principal isotropy subgroups which are neither tori nor discrete groups. Next we obtain an embedding of the Wolf space into a real Grassmannian manifold using twistor sections, which turns out to be a minimal embedding. Finally, we focus our attention on the norm squared $\|s\|^{2}$ of a twistor section $s$. We identify the subset $S_{M}$ where this function attains the maximum value, under a suitable hypothesis. Such sets are classified, and determine totally geodesic submanifolds of the Wolf spaces. Moreover, $\|s\|^{2}$ is a Morse function in the sense of Bott and its critical manifolds consist of the zero locus and $S_{M}$.
\end{abstract}

\section{INTRODUCTION}

The main theorems (Theorems 4.2 and 5.1-5.3) of the present paper concern the zero loci of sections of vector bundles over quaternion-Kähler (QK) manifolds. These are Riemannian manifolds whose linear holonomy groups can be reduced to $\operatorname{Sp}(n) \cdot \operatorname{Sp}(1)$. All known examples of compact quaternion-Kähler manifolds of positive scalar curvature are Riemannian symmetric spaces and Wolf classified compact quaternion symmmetric spaces [23, which are called the Wolf spaces.

The section $s$ in the main theorem satisfies a linear field equation, the twistor equation $\mathcal{D} s=0$. The higher dimensional twistor operator $\mathcal{D}$ is a generalization of the twistor operator in 4-dimensional Riemannian geometry [1], and was defined by Salamon [19] (see Definition 2.1). We also have a generalization of the notion of the anti-self-dual connection, which is called a quaternion ASD connection (Definition 2.2). These can also be defined on a hyper-Kähler (HK) manifold, which, by definition, is a Riemannian manifold of which the linear holonomy group can be reduced to $\operatorname{Sp}(n)$. Both QK and HK manifolds have the canonical quaternion line bundles $\mathbb{H}$ with the induced connections. A section of $V \otimes \mathbb{H}$ that satisfies $\mathcal{D} s=0$ is called a twistor section, where the vector bundle $V$ has an ASD connection. In general, a twistor section $s$ corresponds to a holomorphic section $\tilde{s}$ on the twistor space under the Penrose transform (see for example [7] and 13]).

Received by the editors February 16, 2004.

2000 Mathematics Subject Classification. Primary 53C26.

(C)2008 American Mathematical Society Reverts to public domain 28 years from publication 
Since the zero locus of a holomorphic section is a complex submanifold, it is natural to expect that the zero locus of a twistor section has an inherent rich geometrical structure reflecting the quaternion structure of the ambient space. In fact, the author showed that the zero locus of a twistor section is a QK or an HK submanifold ([16], Theorem 3.2). Then we shall discuss the relation between the zero locus of a twistor section and the zero locus of the corresponding holomorphic section on the twistor space. Naïvely, one may expect that the inverse image of the zero locus of a twistor section by the twistor fibration would be the same as the zero locus of the corresponding section. Although this fails in general, it does hold if we adopt the notion of a real section (Definition 3.6). This reality condition for a section is one of the usual "reality conditions" in the context of the twistor geometry and relates the zero locus of a real twistor section of a homogeneous vector bundle to the real representation of the (covering of the) isometry group. A criterion for the zero locus of a twistor section to be connected is given in cohomological terms (Proposition 3.9). This criterion is exploited in section 4.

In section 4, we are concerned with an irreducible homogeneous vector bundle of the form $V \otimes \mathbb{H}$ on the Wolf space whose rank is less than the dimension of the base manifold. First of all, such vector bundles which admit non-trivial twistor sections are determined (Theorem 4.1). We show that such a bundle necessarily has a twistor section which is transverse to the zero section. Then the zero loci of real and transversal twistor sections of irreducible homogeneous vector bundles on the Wolf spaces are classified (Theorem 4.2). Since the twistor space of the Wolf space is also homogeneous, the Bott-Borel-Weil (BBW) theory implies that the space of the twistor sections is a representation of the isometry group, and the zero locus of a transversal twistor section is connected, together with Proposition 3.9. Combined with Table A in W.C. Hsiang and W.Y. Hsiang [8, we can identify the isometry group of the zero locus, which turns out to be the principal isotropy subgroup of the space of the real twistor sections.

In section 5, our concern is an irreducible homogeneous vector bundle of the form $V \otimes \mathbb{H}$ on the Wolf space whose rank equals the dimension of the base manifold. The cotangent bundle of the Wolf space provides such an example, but it does not admit any non-trivial twistor sections except the cotangent bundle of quaternion projective space $\mathbb{H} P^{n}$ [11]. We can find the other four vector bundles on the complex Grassmannian manifold $G r_{2}\left(\mathbb{C}^{n+2}\right)$ and the real Grassmannian manifold $G r_{4}\left(\mathbb{R}^{12}\right)$ which admit non-trivial twistor sections (see Remark after Theorem 4.1). We classify all the zero loci of real twistor sections of the first three bundles on $\mathbb{H} P^{n}$ and $G r_{2}\left(\mathbb{C}^{n+2}\right)$ (Theorems 5.1 and 5.2) and describe the zero locus of a transversal twistor section of the last two bundles on $G r_{4}\left(\mathbb{R}^{12}\right)$ (Theorem 5.3).

As a result of $\S \S 4$ and 5, there exists a bijection between the zero loci of real and transversal twistor sections of irreducible homogeneous vector bundles on the Wolf spaces and the real representations of simple compact connected Lie groups with non-trivial principal isotropy subgroups which are neither tori nor discrete groups (see "Final conclusion for zero loci" in $\S 5$ ).

In section 6, we consider a mapping from the Wolf space into a real Grassmannian. This mapping is inspired by the well-known Kodaira embedding, which is obtained by holomorphic sections of a (positive) holomorphic line bundle over a Kähler manifold. We may substitute a holomorphic section, a holomorphic bundle and a Kähler manifold into a twistor section, an ASD bundle and a QK manifold, 
respectively. It turns out that a mapping is really an embedding (Theorem 6.1), which we call twistor embedding. Hsiang-Lawson's result [9] implies that a twistor embedding from the Wolf space is a minimal embedding (Theorem 6.2).

In the last section, we focus our attention on a function which is defined by the norm squared of a twistor section. We identify the subset $S_{M}$ where the function attains the maximum value. Here we restrict ourselves to the case that the set of the twistor sections of an irreducible homogeneous vector bundle over the Wolf space is a representation of spherical type (see $\S 7$, for the definition). We can explicitly describe such a subset $S_{M}$ of the function, which is also a totally geodesic submanifold of the Wolf space (Theorem 7.9). In some sense, $S_{M}$ is farthest from the zero locus of the twistor section, both literally and conceptually. Moreover, we can show that the critical submanifolds of the function are only the zero locus and the subset $S_{M}$ (Lemma 7.3). The function turns out to be a Morse function in the sense of Bott (Lemma 7.10).

\section{Preliminaries}

Throughout the paper, all vector bundles are supposed to be complex vector bundles with Hermitian metrics unless otherwise stated.

2.1. Differential operators. We shall define differential operators and an ASD connection on a QK and an HK manifold. By definition, a QK manifold $M$ has a reduced $\operatorname{Sp}(n) \cdot \operatorname{Sp}(1)$ principal bundle $P$ of the orthonormal frame bundle of $M$. Let $\mathbb{H}$ be the standard representation of $\mathrm{Sp}(1)$. In general, a QK manifold $M$ has no lift of $P$ to an $\operatorname{Sp}(n) \times \operatorname{Sp}(1)$ bundle, and so $\mathbb{H}$ does not define an associated vector bundle to $P$ on $M$. Nevertheless, we denote a locally defined associated vector bundle of $\mathbb{H}$ by the same symbol $\mathbb{H}$. In the case of the HK manifold, we obtain $\mathbb{H}$ by considering a lift of a principal $\operatorname{Sp}(n)$ bundle to a principal $\operatorname{Sp}(n) \times \operatorname{Sp}(1)$ bundle, which is the associated bundle of the trivial vector bundle whose global frame is $\{I, J, K\}$. Since the definitions are given in a parallel way for a QK and an HK manifold, we refer to only the case of a QK manifold.

In a similar way, a (locally defined) vector bundle associated with $P$ is denoted by $\mathbb{E}$ of which the typical fibre is the standard representation of $\operatorname{Sp}(n)$. Let $S^{m} \mathbb{H}$ be the $m$ th symmetric power of $\mathbb{H}$ and $\wedge^{i} \mathbb{E}$ be the $i$ th skew-symmetric power of $\mathbb{E}$. From the Clebsch-Gordan formula $S^{m} \mathbb{H} \otimes \mathbb{H} \cong S^{m-1} \mathbb{H} \oplus S^{m+1} \mathbb{H}$, the tensor product $S^{m} \mathbb{H} \otimes \wedge^{i} \mathbb{E} \otimes \mathbb{H} \otimes \mathbb{E}$ involves the two components $S^{m-1} \mathbb{H} \otimes \wedge^{i+1} \mathbb{E}$ and $S^{m+1} \mathbb{H} \otimes \wedge^{i+1} \mathbb{E}$. The projections $p: S^{m} \mathbb{H} \otimes \wedge^{i} \mathbb{E} \otimes \mathbb{H} \otimes \mathbb{E} \rightarrow S^{m-1} \mathbb{H} \otimes \wedge^{i+1} \mathbb{E}$ and $q: S^{m} \mathbb{H} \otimes \wedge^{i} \mathbb{E} \otimes \mathbb{H} \otimes \mathbb{E} \rightarrow S^{m+1} \mathbb{H} \otimes \wedge^{i+1} \mathbb{E}$ determine two types of differential operators on $M[19]$ :

Definition 2.1. The quaternionic operators $D$ and $\mathcal{D}$ are defined by

$$
\begin{aligned}
& D_{m}=p \nabla: S^{m} \mathbb{H} \otimes \wedge^{i} \mathbb{E} \rightarrow S^{m-1} \mathbb{H} \otimes \wedge^{i+1} \mathbb{E}, \\
& \mathcal{D}_{m}=q \nabla: S^{m} \mathbb{H} \otimes \wedge^{i} \mathbb{E} \rightarrow S^{m+1} \mathbb{H} \otimes \wedge^{i+1} \mathbb{E} .
\end{aligned}
$$

Remark. In the case $n=1$, these are the usual Dirac and twistor operators. We shall omit the subscript $m$ when no confusion arises.

2.2. ASD connections. Next, we define an ASD connection.

Definition 2.2 (for example, [13] and 22]). Let $M$ be a QK manifold or an HK manifold and $V$ be a vector bundle with a connection $\nabla$. Then, $\nabla$ is called a 
TABLE 2.1

\begin{tabular}{|l||l|l|l|l|l|l|}
\hline$G$ & $\mathrm{SU}(n)$ & \multicolumn{2}{l|}{$\operatorname{Spin}(n)$} & $\mathrm{Sp}(n)$ \\
\hline$K$ & $\mathrm{U}(1) \operatorname{SU}(n-2)$ & \multicolumn{2}{l|}{$\operatorname{Spin}(n-4)$} & $\operatorname{Sp}(1)$ & $\mathrm{Sp}(n-1)$ \\
\hline & $G$ & $E_{6}$ & $E_{7}$ & $E_{8}$ & $F_{4}$ & $G_{2}$ \\
\hline & $K$ & $\mathrm{SU}(6)$ & $\operatorname{Spin}(12)$ & $E_{7}$ & $\mathrm{Sp}(3)$ & $\mathrm{Sp}(1)$ \\
\cline { 2 - 6 }
\end{tabular}

quaternion $\mathrm{ASD}$ connection if the curvature tensor $R^{\nabla}$ satisfies

$$
R^{\nabla}(I X, I Y)=R^{\nabla}(J X, J Y)=R^{\nabla}(K X, K Y)=R^{\nabla}(X, Y) .
$$

We call $V$ an ASD bundle or instanton bundle if $V$ has a quaternion ASD connection.

Remark. From Galicki-Poon [5], the curvature tensor $R^{\nabla}$ of a quaternion ASD connection satisfies $* R^{\nabla}=\frac{-1}{(2 n-1) !} R^{\nabla} \wedge \Omega^{n-1}$, where $\Omega$ is the fundamental 4-form on $M$ by Bonan [2. Consequently, a quaternion ASD connection gives an example of the anti-self-dual instanton in the sense of Tian 21. In the case of an HK manifold, a quaternion ASD bundle is called a hyperholomorphic vector bundle in Verbitsky [22].

For brevity, a quaternion ASD connection is simply called an ASD connection in this paper.

We can define the coupled quaternion Dirac operator $D$ and the twistor operator $\mathcal{D}$ to sections of a vector bundle $V \otimes S^{m} \mathbb{H} \otimes \wedge^{i} \mathbb{E}$, where $V$ is an ASD bundle. Note that it may happen that there exists a vector bundle $F$ with a connection, which is locally gauge equivalent to a bundle $V \otimes \mathbb{H}$, even if neither an ASD bundle $V$ nor $\mathbb{H}$ is defined globally (e.g., the complexified cotangent bundle $T^{*} M \cong \mathbb{E} \otimes \mathbb{H}$ of a QK manifold). In this paper, such a vector bundle $F$ is also denoted by $V \otimes \mathbb{H}$ with abuse of notation.

2.3. Wolf spaces. The only known examples of compact QK manifolds of positive scalar curvature (positive QK manifolds in the sense of LeBrun and Salamon [12]) are symmetric spaces. Such a symmetric space is called a Wolf space. Wolf classified compact quaternion symmetric spaces [23]. As a result, the (universal covering of the) isometry group of a Wolf space is a maximal compact subgroup of a complex simple connected Lie group and every complex simple connected Lie group corresponds to a Wolf space whose isometry group is the maximal compact subgroup. Hence we use the terminology "the Wolf space of type $A_{n}, B_{n}, \cdots, G_{2}$ ", taking into account the isometry group. (For example, the Wolf space of type $C_{n+1}$ is the quaternion projective space $\mathbb{H} P^{n}$.)

Let $G^{\mathbb{C}}$ be one of the complex simple connected Lie groups and $G$ be the maximal compact subgroup. The corresponding Lie algebras are denoted by $\mathfrak{g}^{\mathbb{C}}$ and $\mathfrak{g}$, respectively. Let $\mathfrak{s p}(1)$ be the subalgebra of $\mathfrak{g}$ generated by the maximal root vector (after we fix a Cartan subalgebra and choose simple roots of $\mathfrak{g}^{\mathbb{C}}$ ). Then, we put $\mathfrak{p}=\mathfrak{k} \oplus \mathfrak{s} \mathfrak{p}(1)$, where $\mathfrak{k}$ is the centraliser of $\mathfrak{s} \mathfrak{p}(1)$ in $\mathfrak{g}$. The corresponding Lie subgroup to $\mathfrak{p}$ is denoted by $P$. Then, the Wolf space is expressed as $G / P$. If we denote by $K$ the corresponding Lie subgroup to $\mathfrak{k}$, then $K$ is concretely written in Table 2.1. 


\section{ZERO LOCUS}

3.1. Known results. We review the results about the zero loci of the twistor sections [16] and present the reason why we consider twistor sections on Wolf spaces.

Definition 3.1. Let $V$ be a vector bundle on a manifold $M$ and let $s$ be a section of $V$. We call a section $s$ non-degenerate if $S=s^{-1}(0)$ is a submanifold of $M$ and the tangent space of $S$ coincides with Ker $d s$, where we regard a section $s$ as a map from an open set $U$ of $M$ to a vector space under a trivialization of $V$ over $U$.

Theorem 3.2. Let $V$ be a (locally defined) ASD bundle over an $H K$ or a $Q K$ manifold $M$. If a section $s$ of $V \otimes \mathbb{H}$ is non-degenerate and satisfies the twistor equation $\mathcal{D} s=0$, then $S$ is an $H K$ or a $Q K$ submanifold, respectively.

If a section of a vector bundle is transverse to the zero section, then the section is non-degenerate. Hence we obtain

Corollary 3.3. Let $V$ be an $A S D$ bundle over an $H K$ or a $Q K$ manifold $M$. If a section s of $V \otimes \mathbb{H}$ is transverse to the zero section and satisfies the twistor equation, then $S$ is an $H K$ or a $Q K$ submanifold, respectively.

3.2. Non-existence. From the theorem of Gray [6], a quaternion submanifold is a totally geodesic submanifold. This implies

Corollary 3.4. Let $V$ be an $A S D$ bundle over an $H K$ or a $Q K$ manifold $M$. If a section $s$ of $V \otimes \mathbb{H}$ is non-degenerate and satisfies the twistor equation, then $S$ is a totally geodesic submanifold of $M$.

We write down the second derivative explicitly.

Theorem 3.5. Let $V$ be an $A S D$ bundle over an $H K$ or a $Q K$ manifold $M$. If a section $s$ of $V \otimes \mathbb{H}$ satisfies the twistor equation, then we have

$$
\nabla^{2} s=-\frac{1}{2} c t s+\operatorname{Sym}^{0} \nabla^{2} s+\frac{1}{2} R s
$$

where $c$ is a positive constant depending only on the dimension of the manifold $M$, $t$ is the scalar curvature [18, and $\mathrm{Sym}^{0} \nabla^{2} s$ is the traceless symmetric part of $\nabla^{2} s$ which can be regarded as a section of $\mathbb{H} \otimes S^{2} \mathbb{E} \otimes V$.

Proof. Now, the section $\nabla^{2} s$ can be considered as a section of $\mathbb{H} \otimes\left(\wedge^{2} \mathbb{E} \oplus S^{2} \mathbb{E}\right) \otimes V$, because $s$ satisfies the twistor equation, and so $\nabla s$ can be regarded as $D s$, which is a section of $\mathbb{E} \otimes V$. When we divide $\nabla^{2} s$ into a (vector bundle valued) symmetric 2 -tensor and a 2 -form, the symmetric 2-tensor is denoted by $\operatorname{Sym} \nabla^{2} s$. Since the skew-symmetric part of $\nabla^{2} s$ is the curvature, we obtain

$$
\nabla^{2} s=\operatorname{Sym} \nabla^{2} s+\frac{1}{2} R s,
$$

where $R$ is the curvature 2-form of $V \otimes \mathbb{H}$. The $\mathbb{H} \otimes \wedge^{2} \mathbb{E} \otimes V$ part of $\nabla^{2} s$ is identified with $\omega_{\mathbb{H}} \otimes \nabla D s$. This corresponds to $\omega_{\mathbb{H}} \otimes \mathcal{D} D s$, by the definition of the twistor operator. Then the formula $D \mathcal{D}+\frac{3}{2} \mathcal{D} D=6 c t \cdot \otimes \omega_{\mathbb{E}}$ [17] and the twistor equation imply that $\mathcal{D} D s=4 c t s \otimes \omega_{\mathbb{E}}$.

However, if we adopt the orthogonal projection when defining the quaternion Dirac operator and the twistor operator, then the coefficient $4 c t$ must be changed. Here the orthogonal projection of $h_{1} \otimes h_{2}$ to $\wedge^{2} \mathbb{H}$ should be $\frac{1}{2} h_{1} \wedge h_{2}$, where $\left\{h_{1}, h_{2}\right\}$ is the standard basis. Similar care is needed when considering the orthogonal 
projection of $\mathbb{E} \otimes \mathbb{E}$ to $\wedge^{2} \mathbb{E}$. Hence the coefficient must be multiplied by $\frac{1}{4}$, and so the $\mathbb{H} \otimes \wedge^{2} E \otimes V$ part of $\nabla^{2} s$ is equal to cts $\otimes \omega_{\mathbb{H}} \otimes \omega_{\mathbb{E}}$.

Next, we divide the $\mathbb{H} \otimes S^{2} \mathbb{E} \otimes V$ part of $\nabla^{2} s$ into a symmetric 2-tensor and a 2 -form. Then the symmetric 2-tensor is trace-free and we denote it by $\operatorname{Sym}^{0} \nabla^{2} s$. We would like to specify $\operatorname{Sym} \nabla^{2} s-\operatorname{Sym}^{0} \nabla^{2} s$, which is the $\mathbb{H} \otimes \wedge^{2} E \otimes V$ component of $\operatorname{Sym} \nabla^{2} s$. The curvature of $\mathbb{H}$ has no component of $S^{2} \mathbb{E}$. Consequently, $\frac{1}{2}\left(R_{V} \otimes I d_{\mathbb{H}}\right) s$ is the $\mathbb{H} \otimes S^{2} \mathbb{E} \otimes V$ component of the skew-symmetric part of $\nabla^{2} s$, where $R_{V}$ is the curvature 2 -form of $V$ and $I d_{\mathbb{H}}$ is the identity transformation of $\mathbb{H}$. Combining this observation with the above result, we obtain

$$
\left(\operatorname{Sym} \nabla^{2} s-\operatorname{Sym}^{0} \nabla^{2} s\right)+\frac{1}{2}\left(I d_{V} \otimes R_{\mathbb{H}}\right) s=c t s \otimes \omega_{\mathbb{E}},
$$

where $R_{\mathbb{H}}$ is the curvature 2 -form of $\mathbb{H}$ and $I d_{V}$ is the identity transformation of $V$. From Proposition 3.2 of [18, we know that $\left(I d_{V} \otimes R_{\mathbb{H}}\right) s=3 c t s$. Therefore, we obtain

$$
\operatorname{Sym} \nabla^{2} s-\operatorname{Sym}^{0} \nabla^{2} s=-\frac{1}{2} c t s .
$$

Theorem 3.5 imposes a restriction on the existence of a twistor section.

Theorem 3.6. On a compact HK manifold, a twistor section is equivalent to a parallel section. On a compact QK manifold of negative scalar curvature, only a zero section is a twistor section.

Proof. We denote the rough Laplacian for a section $s$ of $V \otimes \mathbb{H}$ by $\triangle s=$ $-\sum \nabla^{2} s\left(E_{i}, E_{i}\right)$, where $\left\{E_{1}, \ldots, E_{4 n}\right\}$ is an orthonormal basis. If $s$ is a twistor section, then Theorem 3.5 implies that $\triangle s=2$ ncts. Let $h$ be a Hermitian metric on $V \otimes \mathbb{H}$. From the formula $h(\triangle s, s)+h(s, \triangle s)=\triangle|s|^{2}+2|\nabla s|^{2}$ and the divergence theorem, we obtain

$$
\text { 4nct } \int|s|^{2}=2 \int|\nabla s|^{2} .
$$

On a compact HK manifold, since the scalar curvature vanishes, the left-hand side (LHS) of (10) vanishes.

On a compact QK manifold of negative scalar curvature, the LHS of (1) is nonpositive and the RHS is non-negative, and so both must be zero.

Remark. The above theorem reveals the reason why we consider twistor sections on Wolf spaces.

Next we would like to consider a section of a vector bundle on the twistor space $Z$. The pull-back bundle on the twistor space has a holomorphic structure induced by the pull-back connection if the original connection is ASD. Hence we do not distinguish ASD bundles on an HK or a QK manifold from the pull-back bundles on the twistor space, and we use the same symbol for both. Using the Penrose transform, we can identify the solution space of the twistor equation with the space of holomorphic sections on the twistor space. In our notation, $\{s \in \Gamma(V \otimes \mathbb{H}) \mid$ $\mathcal{D} s=0\} \cong H^{0}(Z, V \otimes \mathcal{O}(1))$, where $V$ in the right-hand side is the pull-back bundle and the line bundle $\mathcal{O}(1)$ corresponds to Salamon's $L$ [18.

Definition 3.7. Let $V$ be an ASD bundle with a quaternion structure over an HK or a QK manifold. The real structure $\tau$ of $V \otimes \mathbb{H}$ is induced by the quaternion structures of $V$ and $\mathbb{H}$. Then a section $s$ of $V \otimes \mathbb{H}$ is called a real section if $s$ can be 
regarded as a section of the real vector bundle $(V \otimes \mathbb{H})^{\mathbb{R}}$, which is the $\tau$-invariant (real) subbundle of $V \otimes \mathbb{H}$. If a holomorphic section $\tilde{s}$ of $V \otimes \mathcal{O}(1)$ corresponds to a real section of $V \otimes \mathbb{H}$ under the Penrose transform, $\tilde{s}$ is also called a real section.

Proposition $3.8([16)$. Let $V$ be an ASD bundle with a quaternion structure over an $H K$ or a $Q K$ manifold $M$. If a holomorphic section $\tilde{s}$ of $V \otimes \mathcal{O}(1)$ is real and nondegenerate, then $\tilde{S}$ is the twistor space of an HK or a QK submanifold $S:=s^{-1}(0)$ of $M$, respectively, where $s$ is the section of $V \otimes \mathbb{H}$ corresponding to $\tilde{s}$ under the Penrose transform.

If a holomorphic section $\tilde{s}$ of $V \otimes \mathcal{O}(1)$ is real and transverse to the zero section, then the corresponding twistor section $s$ is transverse to the zero section of the real vector bundle $(V \otimes \mathbb{H})^{\mathbb{R}}$ over $M$.

Proposition 3.9 ([16]). Let $V$ be an ASD bundle of rank $2 r$ with a quaternion structure over a compact $Q K$ manifold $M$. Suppose that a holomorphic section $\tilde{s}$ of $V \otimes \mathcal{O}(1)$ on the twistor space $Z$ is real and transverse to the zero section. The zero locus $s^{-1}(0)$ is denoted by $S$ where $s$ is the section of $V \otimes \mathbb{H}$ corresponding to $\tilde{s}$ under the Penrose transform. If all the cohomology groups $H^{q}\left(Z, \wedge^{k} V^{*} \otimes \mathcal{O}(-k)\right)$ vanish for $q=0,1, \ldots, 2 n+1$ and $k=1,2, \ldots, 2 r$, then $S$ is connected.

We also need to consider an ASD bundle $V$ over an HK or a QK manifold which has no quaternion structure. Though, in general, we cannot expect that $V \otimes \mathbb{H}$ has an appropriate real structure, the vector bundle $\left(V \oplus V^{*}\right) \otimes \mathbb{H}$ does have a real structure induced by the canonical quaternion structure of $V \oplus V^{*}$, arising from the hermitian structure on $V$. Note that the real vector bundle $\left\{\left(V \oplus V^{*}\right) \otimes \mathbb{H}\right\}^{\mathbb{R}}$ has a complex structure and is then isomorphic to $V \otimes \mathbb{H}$ as a complex vector bundle. Hence a section $s$ of $V \otimes \mathbb{H}$ is identified with a section $(s, \sigma(s))$ of $\left\{\left(V \oplus V^{*}\right) \otimes \mathbb{H}\right\}^{\mathbb{R}}$, where $\sigma$ is the induced real structure. From now on, the section $s$ of $V \otimes \mathbb{H}$ may be regarded as a real section under the identification.

Proposition 3.10 ([16]). Let $V$ be an $A S D$ bundle over an $H K$ or a $Q K$ manifold $M$. If a holomorphic section $\tilde{s}=\left(\tilde{s}_{1}, \widetilde{\sigma\left(s_{1}\right)}\right)$ of $\left(V \oplus V^{*}\right) \otimes \mathcal{O}(1)$ is real and nondegenerate, then $\tilde{S}:=\tilde{s}^{-1}(0)$ is the twistor space of an $H K$ or a $Q K$ submanifold $S_{1}:=s_{1}^{-1}(0)$ of $M$, respectively, where $s_{1}$ is the corresponding section of $V \otimes \mathbb{H}$ to $\tilde{s}_{1}$ under the Penrose transform.

Remark. We can also obtain a similar result to Proposition 3.9 about connectivity of the zero locus of the real and transversal twistor section of $V \otimes \mathbb{H}$. In short, if $H^{q}\left(Z, \wedge^{k}\left(V \oplus V^{*}\right) \otimes \mathcal{O}(-k)\right)$ vanish for $q=0,1, \ldots, 2 n+1$ and $k=1,2, \ldots, 2 r$, then the zero locus is connected, where $r$ is the (complex) rank of $V$. We shall also refer to the result as Proposition 3.9.

\section{Application I}

Since any Wolf space is homogeneous, we can consider a homogeneous vector bundle with the canonical connection. To express a complex homogeneous vector bundle $F$ which is of the form $V \otimes \mathbb{H}$ on the Wolf space $G / P$, we identify such a vector bundle $F=G \times_{K \operatorname{Sp}(1)} V_{0} \otimes \mathbb{H}$ with the representation space $V_{0} \otimes \mathbb{H}$ of $K \mathrm{Sp}(1)$. In particular, if $F$ is supposed to be irreducible, then $V_{0}$ is irreducible as a representation space of $K$. Moreover, if $V_{0}$ has an invariant quaternion structure, then $V_{0} \otimes \mathbb{H}$ has an induced invariant real structure and so we obtain the real vector 
TABLE 4.1

\begin{tabular}{|l||c|c|c|c|c|c|}
\hline$G$ & $\mathrm{SU}(n)$ & $\operatorname{Spin}(n)$ & $\operatorname{Spin}(7)$ & $\operatorname{Spin}(9)$ & \multicolumn{1}{|c|}{$\operatorname{Sp}(n)$} \\
\hline$V_{0}$ & $\mathbb{C}, \mathbb{C}^{*}$ & $\mathbb{C}_{\mathbb{H}}^{2}$ & $S_{\mathbb{H}}$ & \multicolumn{1}{|c|}{$S_{\mathbb{H}}$} & \multicolumn{2}{|c|}{$\mathbb{C}$} \\
\hline$G$ & $\operatorname{Spin}(8)$ & \multicolumn{1}{|c|}{$\operatorname{spin}(10)$} & $E_{6}$ & $E_{7}$ & $F_{4}$ & $G_{2}$ \\
\hline$V_{0}$ & $S_{\mathbb{H}}^{+}, S_{\mathbb{H}}^{-}$ & $S^{+}, S^{-}$ & $\mathbb{C}^{6}, \mathbb{C}^{6^{*}}$ & $\mathbb{C}^{12}$ & $\mathbb{C}_{\mathbb{H}}^{6}$ & $\mathbb{C}_{\mathbb{H}}^{2}$ \\
\hline
\end{tabular}

bundle $\left(V_{0} \otimes \mathbb{H}\right)^{\mathbb{R}}$. In such a case, we adopt the convention that $V_{0} \otimes \mathbb{H}$ represents a real vector bundle $\left(V_{0} \otimes \mathbb{H}\right)^{\mathbb{R}}$.

We can find all irreducible homogeneous vector bundles of the form $V_{0} \otimes \mathbb{H}$ whose (real) ranks are less than the dimension of the base spaces using Lemma 3.3 in 15. In particular, irreducible homogeneous vector bundles which are of the form $V_{0} \otimes \mathbb{H}$ on all the Wolf spaces can be determined by the specified highest weights. Applying the Bott-Borel-Weil (BBW) theorem ([3] and [10]) on the twistor space, we can classify such vector bundles which admit non-trivial twistor sections.

Theorem 4.1. Let $F$ be an irreducible homogeneous vector bundle identified with $V_{0} \otimes \mathbb{H}$ on a Wolf space. If the (real) rank of $F$ is less than the dimension of the Wolf space and admits a non-trivial twistor section, then $F$ is one of the entries in Table 4.1, where, for example, $\mathbb{C}_{\mathbb{H}}^{2}$ means that the representation $\mathbb{C}^{2}$ with an invariant quaternion structure.

More precisely, in the cases of $\mathrm{SU}(n)$ and $\operatorname{Spin}(n), F$ is nothing but the tautological vector bundle (and its dual) on $G r_{2}\left(\mathbb{C}^{n}\right)$ and $G r_{4}\left(\mathbb{R}^{n}\right)$, respectively. In the case of $\operatorname{Sp}(n), \mathbb{C}$ is the trivial representation and $F$ is the tautological quaternion line bundle $\mathbb{H}$ on the quaternion projective space $\mathbb{H} P^{n-1}$. The representation $S\left(S^{+}, S^{-}\right)$is the (half) spin representation of the $K$ factor. In the exceptional cases, the representations are the standard representations of $K$.

Remark. If a vector bundle whose rank equals the dimension of the base manifold is taken into account, the cotangent vector bundle of each Wolf space provides an example, which is of the form $\mathbb{E} \otimes \mathbb{H}$. However, it is shown that the cotangent vector bundles have no non-trivial twistor sections on positive QK manifolds except for $\mathbb{H} P^{n}$ (see LeBrun [11]).

Apart from the cotangent bundle of $\mathbb{H} P^{n}$, the other four irreducible homogeneous vector bundles $V_{0} \otimes \mathbb{H}$ whose (real) ranks are the same as the dimension of the base manifolds admit non-trivial twistor sections.

Let $S$ be the tautological vector bundle on the complex Grassmannian manifold $G r_{2}\left(\mathbb{C}^{n+2}\right)$. (In Theorem 4.1, $S$ corresponds to $\mathbb{C} \otimes \mathbb{H}$.) Since $S$ is a subbundle of the trivial vector bundle $\underline{\mathbb{C}^{n+2}}$ of rank $n+2$, we can consider the quotient bundle $Q$. The canonical connection on $Q$ is ASD [14. Consequently, $Q \otimes S$ is an irreducible homogeneous vector bundle of the form $V_{0} \otimes \mathbb{H}$ and is of complex rank $2 n$. Of course, its dual $Q^{*} \otimes S^{*}$ is also such a vector bundle. It follows from BBW that both $Q \otimes S$ and $Q^{*} \otimes S^{*}$ admit non-trivial twistor sections.

The remaining two examples are constructed on the Wolf space of type Spin(12), where $K$ is $\operatorname{Spin}(8) \operatorname{Sp}(1)$. Let $S^{+}$be the half spin representation of $\operatorname{Spin}(8)$. Then the vector bundle $S^{+} \otimes \mathbb{H}$ is of (complex) rank 16, which is half of the dimension of the base manifold. Of course, using the other half spin representation $S^{-}$, we obtain the desired vector bundle $S^{-} \otimes \mathbb{H}$. We can also check that both $S^{+} \otimes \mathbb{H}$ and $S^{-} \otimes \mathbb{H}$ admit non-trivial twistor sections by BBW. 
TABLE 4.2

\begin{tabular}{|c||c|c|c|c|c|c|c|}
\cline { 2 - 8 } \multicolumn{1}{c|}{} & $G$ & $\mathrm{SU}(n)$ & $\operatorname{Spin}(n)$ & $\operatorname{Spin}(7)$ & $\operatorname{Spin}(9)$ & $\mathrm{Sp}(n)$ \\
\cline { 2 - 8 } \multicolumn{1}{c|}{} & $S$ & $\mathrm{SU}(n-1)$ & $\operatorname{Spin}(n-1)$ & $G_{2}$ & $\operatorname{Spin}(7)$ & $\operatorname{Sp}(n-1)$ \\
\hline$G$ & & $\operatorname{Spin}(8)$ & $\operatorname{Spin}(10)$ & $E_{6}$ & $E_{7}$ & $F_{4}$ & $G_{2}$ \\
\hline$S$ & $\operatorname{Spin}(7), \operatorname{Spin}(7)$ & $\operatorname{Spin}(6)$ & $\operatorname{Spin}(8)$ & $\operatorname{Spin}(8)$ & $\operatorname{Spin}(8)$ & $\mathrm{SU}(3)$ \\
\hline
\end{tabular}

Next, we introduce the main theorem:

Theorem 4.2. Let $F$ be an irreducible homogeneous vector bundle on a Wolf space arising from Theorem 4.1. Then $F$ has a transversal twistor section. The zero loci of such sections are classified in Table 4.2. Here, the zero loci $S$ are also Wolf spaces and so, for example, $S=\mathrm{SU}(n-1)$ means that $S$ is the Wolf space of type $\mathrm{SU}(n-1)$.

Proof. When $V_{0}$ has a quaternion structure, $s \in \Gamma\left(\left(V_{0} \otimes \mathbb{H}\right)^{\mathbb{R}}\right)$ is a real and transversal twistor section if and only if the corresponding holomorphic section $\tilde{s} \in H^{0}\left(V_{0} \otimes \mathcal{O}(1)\right)^{\mathbb{R}}$ under the Penrose transform is transverse to the zero section. In our cases, since every holomorphic vector bundle of the form $V_{0} \otimes \mathcal{O}(1)$ is generated by global sections by the Bott-Borel-Weil (BBW) Theorem ( $[3]$ and [10]), $W=H^{0}\left(V_{0} \otimes \mathcal{O}(1)\right) \rightarrow V_{0} \otimes \mathcal{O}(1)$ is surjective, where $W$ is a trivial bundle with a fibre $H^{0}\left(V_{0} \otimes \mathcal{O}(1)\right)$ on the twistor space. Combined with the $\mathrm{Sp}(1)$-action and the Penrose transform, we obtain that $W \rightarrow V_{0} \otimes \mathbb{H}$ is also surjective, where $W$ is a trivial bundle on a Wolf space whose fiber is considered as the space of all twistor sections. Then, $W^{\mathbb{R}} \rightarrow\left(V_{0} \otimes \mathbb{H}\right)^{\mathbb{R}}$ is also surjective, because the real structures of $W$ and $V_{0} \otimes \mathbb{H}$ are $G$-invariant and $W \rightarrow V_{0} \otimes \mathbb{H}$ preserves the real structures. Hence, by Sard's theorem, the subset consisting of transversal sections is open and dense in the space of all real twistor sections of $F=\left(V_{0} \otimes \mathbb{H}\right)^{\mathbb{R}}$.

When $V_{0}$ does not have any quaternion structures, we consider the vector bundle $\left(V_{0} \oplus V_{0}^{*}\right) \otimes \mathbb{H}$ which has an induced real structure $\sigma$ (Proposition 3.9). Then $s \in \Gamma\left(V_{0} \otimes \mathbb{H}\right)$ is a transversal twistor section if and only if the corresponding real twistor section $(s, \sigma(s))$ of $\left\{\left(V_{0} \otimes V_{0}^{*}\right) \otimes \mathbb{H}\right\}^{\mathbb{R}}$ is transverse to the zero section. Using the previous argument again, we deduce that the subset consisting of transversal sections is open and dense in the space of all twistor sections of $F=V_{0} \otimes \mathbb{H}$. By symmetry, the zero locus of a transversal twistor section of $V_{0} \otimes \mathbb{H}$ is isomorphic as a submanifold to the zero locus of a transversal twistor section of $V_{0}^{*} \otimes \mathbb{H}$.

The BBW theorem implies that $H^{0}\left(V_{0} \otimes \mathcal{O}(1)\right)$ is an irreducible representation of $G$. We need to consider real representations. When $V_{0}$ has a quaternion sturucture, we put $W^{\mathbb{R}}=\left\{H^{0}\left(V_{0} \otimes \mathcal{O}(1)\right)\right\}^{\mathbb{R}}$. Otherwise, we put $W^{\mathbb{R}}=$ $\left\{H^{0}\left(\left(V_{0} \oplus V_{0}^{*}\right) \otimes \mathcal{O}(1)\right)\right\}^{\mathbb{R}}$. (See Table 4.3.) (Here we use Lie algebra terminology. The fundamental weight $\varpi_{i}$ represents the complex representation space with the highest weight $\varpi_{i}$. We number the fundamental weights as in Bourbaki [4.)

In all cases, the real representations $W^{\mathbb{R}}$ of $G$ are irreducible. It is known that the union of all principal orbits is open and dense in the representation space. Therefore a point in the principal orbit of $W^{\mathbb{R}}$ corresponds to a transversal twistor 
TABLE 4.3

\begin{tabular}{l||c||c|c|c|c|c|c|c|}
\hline$G$ & $\operatorname{SU}(n)$ & $\operatorname{Spin}(n)$ & $\operatorname{Spin}(7)$ & $\operatorname{Spin}(9)$ & \multicolumn{2}{|c|}{$\operatorname{Sp}(n)$} \\
\hline$V_{0}$ & $\mathbb{C}, \mathbb{C}^{*}$ & $\mathbb{C}_{\mathbb{H}}^{2}$ & $S_{\mathbb{H}}$ & $S_{\mathbb{H}}$ & \multicolumn{2}{|c|}{$\mathbb{C}$} \\
\hline$W^{\mathbb{R}}$ & $\left(\mathbb{C}^{n} \oplus \mathbb{C}^{n^{*}}\right)^{\mathbb{R}}$ & $\mathbb{R}^{n}$ & $S^{\mathbb{R}}$ & $S^{\mathbb{R}}$ & $\left(\mathbb{H}^{n} \oplus \mathbb{H}^{n}\right)^{\mathbb{R}}$ \\
\hline$G$ & $\operatorname{Spin}(8)$ & $\operatorname{Spin}(10)$ & $E_{6}$ & $E_{7}$ & $F_{4}$ & $G_{2}$ \\
\hline$V_{0}$ & $S_{\mathbb{H}}^{+}, S_{\mathbb{H}}^{-}$ & $S^{+}, S^{-}$ & $\mathbb{C}^{6}, \mathbb{C}^{6^{*}}$ & $\mathbb{C}^{12}$ & $\mathbb{C}_{\mathbb{H}}^{6}$ & $\mathbb{C}_{\mathbb{H}}^{2}$ \\
\hline$W^{\mathbb{R}}$ & $S^{+^{\mathbb{R}}}, S^{-\mathbb{R}}$ & $\left(S^{+} \oplus S^{-}\right)^{\mathbb{R}}$ & $\left(\varpi_{1}+\varpi_{6}\right)^{\mathbb{R}}$ & $\left(\varpi_{7}+\varpi_{7}\right)^{\mathbb{R}}$ & $\varpi_{4}^{\mathbb{R}}$ & $\varpi_{1}^{\mathbb{R}}$ \\
\hline
\end{tabular}

section, because the transversality is invariant under the $G$-action. We pick up such a real and transversal twistor section $s$ in $W^{\mathbb{R}}$ and denote by $H$ the isotropy subgroup of $G$ at $s$. Then $H$ is determined by W.C. Hsiang and W.Y. Hsiang 8 , Table A]. Let $S$ be the zero locus of $s$. Since $s$ is a transversal section, Corollary 3.3 yields that $S$ is a compact QK submanifold on which $H$ acts, and we can know the dimension of $S$.

Finally, we use Proposition 3.9 to show the connectivity of $S$. Since $F$ is homogeneous, the vector bundles $\wedge^{k} V_{0} \otimes \mathcal{O}(-k)$ and $\wedge^{k}\left(V_{0} \oplus V_{0}^{*}\right) \otimes \mathcal{O}(-k)$ are also homogeneous but usually not irreducible. Then the BBW theorem with involved irreducible decompositions of the homogeneous bundles implies that all relevant cohomology groups vanish (though the procedure is tedious and long). Consequently, $S$ is connected and so, $S$ is also a Wolf space, because $S$ is a totally geodesic submanifold of the original Wolf space (Corollary 3.4). We can check that $H$ acts non-trivially on $S$. Wolf's classification implies that $S$ is the Wolf space of type $H$.

Remark. The Wolf space of type $\operatorname{Spin}(7)\left(G r_{4}\left(\mathbb{R}^{7}\right)\right)$ appears three times in Table 4.2 as a submanifold of the Wolf space of type $\operatorname{Spin}(8)\left(G r_{4}\left(\mathbb{R}^{8}\right)\right)$. In our situation, we can compute the Poincaré dual of the homology class represented by the submanifold $G r_{4}\left(\mathbb{R}^{7}\right)$ as the top Chern class of the vector bundle. As a result, three copies of $G r_{4}\left(\mathbb{R}^{7}\right)$ represent different homology classes (Tasaki showed this fact from the viewpoint of calibrated geometry [20]).

\section{Application II}

In this section, we identify the zero loci of non-trivial twistor sections of irreducible homogeneous vector bundles of which the ranks are equal to the dimension of the base spaces. Such vector bundles are classified in the Remark after Theorem 4.1 .

First, we determine zero loci of all (real) twistor 1-forms on $\mathbb{H} P^{n}$. From our definition of a real section, a real 1-form is a 1-form that is real in the usual sense, and so the term "real" is omitted.

Theorem 5.1. The zero locus of a twistor 1-form on $\mathbb{H} P^{n}$ is of the form $\coprod_{p=1}^{l} \mathbb{H} P^{k_{p}}$, where $\mathbb{H} P^{k_{p}}=\mathbb{P}\left(\mathbb{H}^{k_{p}+1}\right), \bigoplus_{p=1}^{l} \mathbb{H}^{k_{p}+1}=\mathbb{H}^{n+1}$ and $\bigoplus$ means the orthogonal direct sum of quaternion vector subspaces $\mathbb{H}^{k_{p}+1}(p=1, \ldots, l)$ of $\mathbb{H}^{n+1}$. The numbers $k_{p}$ and $l$ depend on the twistor 1 -form.

Proof. By the Bott-Borel-Weil theorem (3] and [10]), $H^{0}\left(\mathbb{C} P^{2 n+1}, \mathbb{E} \otimes \mathcal{O}(1)\right)$ is identified with the $\operatorname{Sp}(n+1)$ representation space $\wedge_{0}^{2} \mathbb{C}^{2 n+2}$, where $\wedge_{0}^{2} \mathbb{C}^{2 n+2}$ is the orthogonal complement of $\omega$ in $\wedge^{2} \mathbb{C}^{2 n+2}$, which is the invariant symplectic form on 
the standard representation $\mathbb{C}^{2 n+2}$ of $\operatorname{Sp}(n+1)$. Since the real structure is $\operatorname{Sp}(n+1)$ invariant from our definition, Schur's lemma implies that the set of real sections corresponds to the real subspace $\left(\wedge_{0}^{2} \mathbb{C}^{2 n+2}\right)^{\mathbb{R}}$ invariant by the standard $\operatorname{Sp}(n+1)$ invariant real structure $\sigma$. Note that $\sigma$ is induced by the restriction of $j \otimes j$, where $j$ is the quaternion structure of $\mathbb{C}^{2 n+2}$. Using $\omega,\left(\wedge_{0}^{2} \mathbb{C}^{2 n+2}\right)^{\mathbb{R}}$ is identified with the space of quaternion Hermitian matrices. If we fix $\phi \in \wedge_{0}^{2}\left(\mathbb{C}^{2 n+2}\right)^{\mathbb{R}}$ and regard $\phi$ as a quaternion Hermitian matrix, then we obtain an orthogonal decomposition of $\mathbb{C}^{2 n+2} \cong \mathbb{H}^{n+1}$ :

$$
\mathbb{H}^{n+1}=\bigoplus_{p=1}^{l} \mathbb{H}^{k_{p}+1},
$$

where $\mathbb{H}^{k_{p}+1}(p=1, \ldots, l)$ are the eigenspaces of $\phi$.

We take a compatible unitary basis $e_{1}, e_{2}, \ldots, e_{2 n+2}$ of $\mathbb{C}^{2 n+2}$ with the quaternion structure $\left(\omega=e_{1} \wedge e_{2}+\cdots+e_{2 n+1} \wedge e_{2 n+2}\right)$. To express $\mathbb{H} P^{n}$ as a homogeneous space, the isotropy subgroup of $\operatorname{Sp}(n+1)$ at the quaternion line $\mathbb{H} e_{1} \subset \mathbb{C}^{2 n+2}$ is denoted by $\operatorname{Sp}(1) \times \operatorname{Sp}(n)$. The twistor space $\mathbb{C} P^{2 n+1}$ is written as $\operatorname{Sp}(n+1) / \mathrm{U}(1) \times \operatorname{Sp}(n)$, where $\mathrm{U}(1)$ is the standard subgroup of $\operatorname{Sp}(1)$. Let $\pi_{2}: \wedge_{0}^{2} \mathbb{C}^{2 n+2} \longrightarrow \mathbb{C}^{2 n}$ be the orthogonal projection, where $\mathbb{C}^{2 n}$ is spanned by elements $e_{2} \wedge e_{q} \in \wedge_{0}^{2} \mathbb{C}^{2 n+2}$, $q=3,4, \ldots, 2 n+2$. Then the homogeneous holomorphic vector bundle $\mathbb{E} \otimes \mathcal{O}(1)$ is expressed as $\operatorname{Sp}(n+1) \times{ }_{\mathrm{U}(1) \times \mathrm{Sp}(n)} \mathbb{C}^{2 n}$. The BBW theorem yields that $\left[g, \pi_{2}\left(g^{-1} \cdot \psi\right)\right]$ is a holomorphic section of $\mathbb{E} \otimes \mathcal{O}(1)$, where $g \in \operatorname{Sp}(n+1)$ and $\psi \in \wedge_{0}^{2} \mathbb{C}^{2 n+2}$.

Let $\pi_{1}: \wedge_{0}^{2} \mathbb{C}^{2 n+2} \longrightarrow \mathbb{C}^{2 n^{*}}$ be the orthogonal projection, where $\mathbb{C}^{2 n^{*}}$ is spanned by elements $e_{1} \wedge e_{q} \in \wedge_{0}^{2} \mathbb{C}^{2 n+2}, q=3,4, \ldots, 2 n+2$. When $h$ denotes an invariant Hermitian inner product on $\wedge_{0}^{2} \mathbb{C}^{2 n+2}, h$ satisfies

$$
h\left(\sigma\left(\psi_{1}\right), \sigma\left(\psi_{2}\right)\right)=\overline{h\left(\psi_{1}, \psi_{2}\right)},
$$

for any $\psi_{1}, \psi_{2} \in \wedge_{0}^{2} \mathbb{C}^{2 n+2}$. If $\phi \in\left(\wedge_{0}^{2} \mathbb{C}^{2 n+2}\right)^{\mathbb{R}}$, then (2) implies that the condition $\pi_{2}\left(g^{-1} \cdot \phi\right)=0$ is equivalent to $\pi_{1}\left(g^{-1} \cdot \phi\right)=0$. Hence, we define the orthogonal projection $\pi: \wedge_{0}^{2} \mathbb{C}^{2 n+2} \longrightarrow \mathbb{C}^{2} \otimes \mathbb{C}^{2 n}$, where $\mathbb{C}^{2} \otimes \mathbb{C}^{2 n}$ is spanned by elements $e_{i} \wedge e_{q} \in \wedge_{0}^{2} \mathbb{C}^{2 n+2}, i=1,2$ and $q=3,4, \ldots, 2 n+2$. Then the zero locus of the section defined by $\phi$ is

$$
\tilde{S}=\left\{[g]=\left[g e_{1}\right] \in \mathbb{C} P^{2 n+1} \mid \pi\left(g^{-1} \cdot \phi\right)=0\right\} .
$$

On the other hand, it is easy to show that $\pi \circ \sigma=\sigma \circ \pi$. Consequently, if $\phi \in\left(\wedge_{0}^{2} \mathbb{C}^{2 n+2}\right)^{\mathbb{R}}$, then $\pi\left(g^{-1} \cdot \phi\right) \in\left(\mathbb{C}^{2} \otimes \mathbb{C}^{2 n}\right)^{\mathbb{R}}$, where $\left(\mathbb{C}^{2} \otimes \mathbb{C}^{2 n}\right)^{\mathbb{R}}$ is the $\sigma$-invariant real subspace of $\mathbb{C}^{2} \otimes \mathbb{C}^{2 n}$. When we regard an element of $\wedge^{2} \mathbb{C}^{2 n+2}$ as a matrix, we have that $h(\xi, \eta)=\operatorname{trace}\left(\xi \eta^{*}\right)=\sum_{A=1}^{2 n+2} h_{\mathbb{C}}\left(\xi e_{A}, \eta e_{A}\right)$, where $h_{\mathbb{C}}$ is an invariant Hermitian inner product on $\mathbb{C}^{2 n+2}$. Hence $\pi\left(g^{-1} \cdot \phi\right)=0$ if and only if there exist complex numbers $\alpha$ and $\beta$ such that $\left(g^{-1} \cdot \phi\right) e_{1}=\alpha e_{1}+\beta e_{2}$. Here note that $\left(g^{-1} \cdot \phi\right)$ can be regarded as a quaternion matrix, so the condition that $\left(g^{-1} \cdot \phi\right) e_{1}=\alpha e_{1}+\beta e_{2}$ implies that $\left(g^{-1} \cdot \phi\right) e_{2}=-\bar{\beta} e_{1}+\bar{\alpha} e_{2}$. Then we obtain $\beta=0$, because $\left(g^{-1} \cdot \phi\right)$ is Hermitian, and so $\beta=h_{\mathbb{C}}\left(\left(g^{-1} \cdot \phi\right) e_{1}, e_{2}\right)=h_{\mathbb{C}}\left(e_{1},\left(g^{-1} \cdot \phi\right) e_{2}\right)=-\beta$. Since an eigenvalue of a Hermitian matrix is real, $\alpha$ is a real number. Consequently we obtain

$$
\begin{aligned}
\tilde{S}=\left\{[g]=\left[g e_{1}\right] \in \mathbb{C} P^{2 n+1} \mid\right. & \left(g^{-1} \cdot \phi\right) e_{1}=a e_{1} \text { and }\left(g^{-1} \cdot \phi\right) e_{2}=a e_{2} \\
& \text { for some real number } a\} .
\end{aligned}
$$


When $\phi$ is regarded as a matrix, the action is defined by $g^{-1} \cdot \phi=g^{-1} \phi g$. It follows that

$$
\tilde{S}=\left\{[g]=\left[g e_{1}\right] \in \mathbb{C} P^{2 n+1} \mid g e_{1} \in \mathbb{H}^{k_{p}+1} \text { for some } p\right\} .
$$

Propositon 3.8 yields the desired result.

Remark. Theorem 5.1 implies that the zero locus of the transversal section is

$$
\{\text { pt. }\} \cup\{\text { pt. }\} \cup \cdots \cup\{\text { pt. }\} \quad(n+1 \text { times }) .
$$

From Hsiang and Hsiang [8], we know that the principal isotropy subgroup is

$$
\operatorname{Sp}(1) \times \operatorname{Sp}(1) \times \cdots \times \operatorname{Sp}(1) \quad(n+1 \text { times }) .
$$

Theorem 5.2. The zero set of a twistor section of $Q \otimes S \rightarrow G r_{2}\left(\mathbb{C}^{n+2}\right)(n>2)$ is a disjoint union of submanifolds $\left(\coprod_{p=1}^{m} \mathbb{H} P^{k_{p}}\right) \amalg G r_{2}\left(\mathbb{C}^{l}\right)$, where $\mathbb{H} P^{k_{p}}=\mathbb{P}\left(\mathbb{H}^{k_{p}+1}\right)$, $\left(\bigoplus_{p=1}^{l} \mathbb{H}^{k_{p}+1}\right) \oplus\left(\mathbb{C}^{l} \oplus \mathbb{C}^{l^{*}}\right)=\mathbb{C}^{n+2} \oplus \mathbb{C}^{n+2^{*}}=\mathbb{H}^{n+2}$ and $\bigoplus$ means the orthogonal direct sum of quaternion vector subspaces $\mathbb{H}^{k_{p}+1}(p=1, \ldots, m)$ and $\mathbb{C}^{l} \oplus \mathbb{C}^{l^{*}}$ of $\mathbb{H}^{n+2}$. When $l \leqq 1, G r_{2}\left(\mathbb{C}^{l}\right)$ means the empty subset. The numbers $k_{p}, m$ and $l$ depend on the twistor section.

Remark. In the case that $n=2$, in other words, on $G r_{2}\left(\mathbb{C}^{4}\right)$, the vector bundle $Q \otimes S$ has an invariant real structure and the isomorphism between $\operatorname{Spin}(6)$ and $\mathrm{SU}(4)$ enables us to identify $(Q \otimes S)^{\mathbb{R}}$ with the tautological vector bundle on $G r_{4}\left(\mathbb{R}^{6}\right)$. Hence the statement reduces to Theorem 4.2. We can easily show that all orbits except the zero orbit corresponds to transversal sections in this case, so only $\mathbb{H} P^{1}$ appears as the zero locus.

Proof. The vector bundle $Q \otimes S$ can be expressed as $V_{0} \otimes \mathbb{H}$. Then $V_{0}$ has no invariant quaternion structure. Consequently, we consider $\left(V_{0} \oplus V_{0}^{*}\right) \otimes \mathbb{H}$ (Proposition 3.12). By BBW, $H^{0}\left(Z,\left(V_{0} \oplus V_{0}^{*}\right) \otimes \mathcal{O}(1)\right)$ is identified with the representation space $\wedge^{2} \mathbb{C}^{n+2} \oplus \wedge^{2} \mathbb{C}^{n+2^{*}}$, which has an invariant real structure induced from the real structure of $\left(V_{0} \oplus V_{0}^{*}\right) \otimes \mathbb{H}$.

We introduce an invariant quaternion structure $j$ of $\mathbb{C}^{n+2} \oplus \mathbb{C}^{n+2^{*}}$. Then we can obtain the invariant real structure of $\wedge^{2}\left(\mathbb{C}^{n+2} \oplus \mathbb{C}^{n+2^{*}}\right)$ induced from the quaternion structure $j$ of $\mathbb{C}^{n+2} \oplus \mathbb{C}^{n+2^{*}}$. It is easy to show that the induced real structure can be restricted to the subspace $\wedge^{2} \mathbb{C}^{n+2} \oplus \wedge^{2} \mathbb{C}^{n+2^{*}}$ of $\wedge^{2}\left(\mathbb{C}^{n+2} \oplus \mathbb{C}^{n+2^{*}}\right)$. Since $\wedge^{2} \mathbb{C}^{n+2}$ and $\wedge^{2} \mathbb{C}^{n+2^{*}}$ have no invariant real structures, a variant of Schur's lemma implies that the two real structures of $\wedge^{2} \mathbb{C}^{n+2} \oplus \wedge^{2} \mathbb{C}^{n+2^{*}}$ coincide with each other.

Next, if $\wedge^{2} \mathbb{C}^{n+2} \oplus \wedge^{2} \mathbb{C}^{n+2^{*}}$ is regarded as a subspace of endomorphisms of $\mathbb{C}^{n+2} \oplus$ $\mathbb{C}^{n+2^{*}}=\mathbb{H}^{n+2}$, then a real twistor section $s \in H^{0}\left(Z,\left(V_{0} \oplus V_{0}^{*}\right) \otimes \mathcal{O}(1)\right)$ can be expressed as an endomorphism:

$$
s=\Phi=\left(\begin{array}{cc}
O & -\bar{A} \\
A & O
\end{array}\right)
$$

where $A$ is a skew-symmetric matrix under a unitary basis $e_{1}, \ldots, e_{n+2}$ of $\mathbb{C}^{n+2}$ and the dual basis $e^{1}, \ldots, e^{n+2}$ of $\mathbb{C}^{n+2^{*}}$ satisfying $e^{k}=j e_{k}$ when $\mathbb{C}^{n+2}$ and $\mathbb{C}^{n+2^{*}}$ are regarded as subspaces of the direct sum. Hence $\Phi$ is a quaternion Hermitian matrix, and we obtain the orthogonal decomposition of $\mathbb{C}^{n+2} \oplus \mathbb{C}^{n+2^{*}}=\mathbb{H}^{n+2}$. 
Since $\Phi$ is of a particular form, the orthogonal decomposition has some features. If we write the corresponding eigenspace to the eigenvalue $a \in \mathbb{R}$ as $\mathbb{H}(a)$, then

$$
\mathbb{H}^{n+2}=\bigoplus_{p=1}^{m}\left\{\mathbb{H}\left(a_{p}\right) \oplus \mathbb{H}\left(-a_{p}\right)\right\} \oplus \mathbb{H}(0),
$$

where the $a_{p}$ 's are positive numbers. Moreover, as a complex vector space, we have other decompositions:

$$
\left\{\mathbb{H}\left(a_{p}\right) \oplus \mathbb{H}\left(-a_{p}\right)\right\}=\mathbb{C}\left(a_{p}\right) \oplus \mathbb{C}\left(a_{p}\right)^{*}
$$

where $\mathbb{C}\left(a_{p}\right)$ (resp. $\left.\mathbb{C}\left(a_{p}\right)^{*}\right)$ is a subspace of $\mathbb{C}^{n+2}$ (resp. $\mathbb{C}^{n+2^{*}}$ ), and

$$
\mathbb{H}(0)=\mathbb{C}(0) \oplus \mathbb{C}(0)^{*}
$$

where $\mathbb{C}(0)$ (resp. $\left.\mathbb{C}(0)^{*}\right)$ is a subspace of $\mathbb{C}^{n+2}$ (resp. $\mathbb{C}^{n+2^{*}}$ ). In both cases, we have $j \mathbb{C}(a)=\mathbb{C}(a)^{*}\left(a=a_{1}, \ldots, a_{m}, 0\right)$. Finally, $\mathbb{C}\left(a_{p}\right)$ and $\mathbb{C}\left(a_{p}\right)^{*}$ have quaternion structures induced from $\Phi$ (or $A$ ) respectively which are compatible with the Hermitian structures up to scale.

We describe the twistor space $Z_{F}$ of $G r_{2}\left(\mathbb{C}^{n+2}\right)$ :

$$
Z_{F}=\left\{\left(\left[g e_{1}\right],\left[g e^{2}\right]\right) \in \mathbb{P}\left(\mathbb{C}^{n+2}\right) \times \mathbb{P}\left(\mathbb{C}^{n+2^{*}}\right) \mid g \in \mathrm{SU}(n+2)\right\} .
$$

The subspace spanned by $e_{3}, \ldots, e_{n+2}\left(\right.$ resp. $\left.e^{3}, \ldots, e^{n+2}\right)$ of $\mathbb{C}^{n+2}\left(\right.$ resp. $\left.\mathbb{C}^{n+2^{*}}\right)$ is denoted by $\mathbb{C}^{n}$ (resp. $\mathbb{C}^{n^{*}}$ ). Let $\pi_{1}: \wedge^{2} \mathbb{C}^{n+2} \rightarrow \mathbb{C}_{2} \otimes \mathbb{C}^{n}$ be the orthogonal projection and $\pi_{2}: \wedge^{2} \mathbb{C}^{n+2^{*}} \rightarrow \mathbb{C} e^{1} \otimes \mathbb{C}^{n^{*}}$ be the orthogonal projection. We use $\pi_{1}$ and $\pi_{2}$ to define the orthogonal projection $\pi=\pi_{1} \oplus \pi_{2}: \wedge^{2} \mathbb{C}^{n+2} \oplus \wedge^{2} \mathbb{C}^{n+2^{*}} \rightarrow$ $\left(\mathbb{C} e_{2} \otimes \mathbb{C}^{n}\right) \oplus\left(\mathbb{C} e^{1} \otimes \mathbb{C}^{n^{*}}\right)$ Then the corresponding section $\tilde{s}$ to $\Phi$ is expressed as

$$
\tilde{s}\left(\left[g e_{1}\right],\left[g e^{2}\right]\right)=\left[g, \pi\left(g^{-1} \cdot \Phi\right)\right] .
$$

The zero locus $\tilde{S}$ of the section $\tilde{s}$ is

$$
\tilde{S}=\left\{[g]=\left(\left[g e_{1}\right],\left[g e^{2}\right]\right) \mid \pi\left(g^{-1} \cdot \Phi\right)=0\right\} .
$$

When we regard $e_{1}, \ldots, e_{n+2}$ and $e^{1}, \ldots, e^{n+2}$ as vectors of the direct sum $\mathbb{C}^{n+2} \oplus$ $\mathbb{C}^{n+2^{*}}$, the definition of $\Phi$ implies that $\pi\left(g^{-1} \cdot \Phi\right)=0$ if and only if

$$
\left(g^{-1} \cdot \Phi\right) e_{1}=\alpha e^{2} \quad \text { and } \quad\left(g^{-1} \cdot \Phi\right) e^{2}=\bar{\alpha} e_{1}
$$

for some complex number $\alpha$. Applying $j$, we also obtain

$$
\left(g^{-1} \cdot \Phi\right) e^{1}=-\bar{\alpha} e_{2} \quad \text { and } \quad\left(g^{-1} \cdot \Phi\right) e_{2}=-\alpha e^{1} .
$$

This is equivalent to the condition:

$$
\begin{gathered}
g e_{1}, g e_{2}, g e^{1}, g e^{2} \in \mathbb{H}(|\alpha|) \oplus \mathbb{H}(-|\alpha|), \\
\left(g e_{1}, g e_{2}, g e^{1}, g e^{2} \in \mathbb{H}(0), \text { if } \alpha=0\right)
\end{gathered}
$$

or equivalently,

$$
g e_{1}, g e_{2} \in \mathbb{C}(|\alpha|), \quad g e^{1}, g e^{2} \in \mathbb{C}(|\alpha|)^{*} .
$$

Consequently, we can determine the zero locus $\tilde{S}$. If $\operatorname{dim}_{\mathbb{H}} \mathbb{H}(0)=0$ or 1 , then

$$
\tilde{S}=\coprod_{p=1}^{l}\left(\mathbb{P}\left(\mathbb{C}\left(a_{p}\right)\right) \times \mathbb{P}\left(\mathbb{C}\left(a_{p}\right)^{*}\right)\right) \cap Z_{F}
$$


If $\operatorname{dim}_{\mathbb{H}} \mathbb{H}(0) \geqq 2$, then

$$
\tilde{S}=\left(\coprod_{p=1}^{l}\left(\mathbb{P}\left(\mathbb{C}\left(a_{p}\right)\right) \times \mathbb{P}\left(\mathbb{C}\left(a_{p}\right)^{*}\right)\right) \amalg\left(\mathbb{P}(\mathbb{C}(0)) \times \mathbb{P}\left(\mathbb{C}(0)^{*}\right)\right)\right) \cap Z_{F} .
$$

The twistor fibration $p: Z_{F} \rightarrow G r\left(\mathbb{C}^{n+2}\right)$ is given by

$$
p\left(\left[g e_{1}\right],\left[g e^{2}\right]\right)=\mathbb{C} g e_{1} \oplus \mathbb{C} g e_{2} .
$$

If $\left(\left[g e_{1}\right],\left[g e^{2}\right]\right) \in \mathbb{P}\left(\mathbb{C}\left(a_{p}\right)\right) \times \mathbb{P}\left(\mathbb{C}\left(a_{p}\right)^{*}\right)$, then $\mathbb{C} g e_{1} \oplus \mathbb{C} g e_{2}$ is a quaternion subspace of $\mathbb{C}\left(a_{p}\right)$, because $\left(\Phi g e_{1}\right)\left(g e_{2}\right)=\alpha \neq 0$, where $\Phi$ is regarded as the induced symplectic form of $\mathbb{C}\left(a_{p}\right)$. Therefore we obtain the desired result.

Remark. Theorem 5.2 implies that the zero locus of the transversal section is

$$
\left\{\begin{array}{lll}
\{\text { pt. }\} \cup\{\text { pt. }\} \cup \cdots \cup\{\text { pt. }\} & \left(\frac{n+2}{2} \text { times }\right) & n \text { even }, \\
\{\text { pt. }\} \cup\{\text { pt. }\} \cup \cdots \cup\{\text { pt. }\} & \left(\frac{n+1}{2} \text { times }\right) & n \text { odd. }
\end{array}\right.
$$

From Hsiang and Hsiang [8], we know that the principal isotropy subgroup is

$$
\left\{\begin{array}{lll}
\mathrm{SU}(2) \times \mathrm{SU}(2) \times \cdots \times \mathrm{SU}(2) & \left(\frac{n+2}{2} \text { times }\right) & n \text { even } \\
\mathrm{SU}(2) \times \mathrm{SU}(2) \times \cdots \times \mathrm{SU}(2) & \left(\frac{n+1}{2} \text { times }\right) & n \text { odd }
\end{array}\right.
$$

Theorem 5.3. The zero locus of a transversal twistor section of $S^{+} \otimes \mathbb{H}$ on $G r_{4}\left(\mathbb{R}^{12}\right)$ (the Wolf space of type $\operatorname{Spin}(12)$ ) consists of three points.

Proof. Since $S^{+}$has no invariant quaternion structure, we deal with $\left(S^{+} \oplus S^{+^{*}}\right) \otimes$ $\mathbb{H} \cong\left(S^{+} \oplus S^{+}\right) \otimes \mathbb{H}$ and the induced real structure (Proposition 3.10). Using the same argument as in the proof of Theorem 4.2, we deduce that $S^{+} \otimes \mathbb{H}$ has a transversal twistor section.

We can use the BBW theorem to compute related cohomology groups $H^{p}\left(Z, \wedge^{k}\left(S^{+} \oplus S^{+}\right) \otimes \mathcal{O}(-k)\right), p=0,1, \ldots, 17$ and $k=1,2, \ldots, 16$. As a result, we obtain

$$
\begin{aligned}
& H^{4}\left(Z, \wedge^{4}\left(S^{+} \oplus S^{+}\right) \otimes \mathcal{O}(-4)\right) \cong \mathbb{C}, \\
& H^{8}\left(Z, \wedge^{8}\left(S^{+} \oplus S^{+}\right) \otimes \mathcal{O}(-8)\right) \cong \mathbb{C},
\end{aligned}
$$

and the other cohomology groups vanish. Let $\tilde{S}$ be the zero locus of a transversal section of $\left(S^{+} \oplus S^{+}\right) \otimes \mathcal{O}(1)$. Proposition 3.7 yields that $\tilde{S}$ consists of several twistor fibres or real lines. In the same way as in the proof of [16, Proposition 3.8], we can show that there exists a spectral sequence such that

$$
E_{1}^{1,0}=H^{0}\left(\tilde{S}, \mathcal{O}_{\tilde{S}}\right), \quad E_{1}^{0,0}=H^{0}\left(Z, \mathcal{O}_{Z}\right) \cong \mathbb{C}, \quad E_{1}^{-4,4}=E_{1}^{-8,8}=\mathbb{C}
$$

and the other $E_{1}$-terms vanish. Applying the spectral sequence, we have three maps

$$
\begin{gathered}
\alpha: H^{0}\left(Z, \mathcal{O}_{Z}\right) \cong \mathbb{C} \rightarrow H^{0}\left(\tilde{S}, \mathcal{O}_{\tilde{S}}\right), \\
\beta: \mathbb{C} \rightarrow \operatorname{Coker} \alpha, \quad \gamma: \mathbb{C} \rightarrow \operatorname{Coker} \alpha / \operatorname{Im} \beta
\end{gathered}
$$

and

$$
\begin{aligned}
& E_{\infty}^{1,0}=(\operatorname{Coker} \alpha / \operatorname{Im} \beta) / \operatorname{Im} \gamma, \quad E_{\infty}^{0,0}=\operatorname{Ker} \alpha \\
& E_{\infty}^{-4,4}=\operatorname{Ker} \beta, \quad E_{\infty}^{-8,8}=\operatorname{Ker} \gamma
\end{aligned}
$$


The spectral sequence abuts to 0 , because of transversality. Consequently, we have

$$
H^{0}\left(\tilde{S}, \mathcal{O}_{\tilde{S}}\right)=\mathbb{C}^{3} .
$$

Hence $\tilde{S}$ consists of three connected components and so, three real lines.

Remark. The BBW theorem implies that

$$
H^{0}\left(Z,\left(S^{+} \oplus S^{+}\right) \otimes \mathcal{O}(1)\right) \cong \mathbb{S}^{+} \oplus \mathbb{S}^{+}
$$

as $\operatorname{Spin}(12)$ modules, where $\mathbb{S}^{+}$denotes the half-spin representation of $\operatorname{Spin}(12)$. In a similar argument in the proof of Theorem 4.1, we show that a point in the principal orbit of $\left(\mathbb{S}^{+} \oplus \mathbb{S}^{+}\right)^{\mathbb{R}}$ corresponds to a real and transversal section of $\left(S^{+} \oplus S^{+}\right) \otimes \mathcal{O}(1)$. Hsiang and Hsiang show that the isotropy group of the principal orbit of $\left(\mathbb{S}^{+} \oplus \mathbb{S}^{+}\right)^{\mathbb{R}}$ is $\mathrm{SU}(2) \times \mathrm{SU}(2) \times \mathrm{SU}(2)[8$, Table $\mathrm{A}]$.

Final conclusion for zero loci. Compared with Table A in [8], we conclude that the zero loci of real and transversal twistor sections of irreducible homogeneous vector bundles on the Wolf spaces $G / P$ correspond in a one-to-one fashion to the real representations with non-trivial principal isotropy subgroups which are neither toral subgroups nor discrete subgroups of $G$. The principal isotropy subgroups are the isometry groups of the zero loci.

\section{TWISTOR EMBEDDING}

Let $F$ be an irreducible homogeneous vector bundle identified with $V_{0} \otimes \mathbb{H}$ on a Wolf space $G / P$ which admits a non-trivial twistor section.

As in the proof of Theorem $4.2, W^{\mathbb{R}}$ denotes the irreducible representation space of $G$ consisting of real twistor sections of $F$. More explicitly, $W^{\mathbb{R}}$ is defined to be

$$
\left\{\begin{array}{l}
\left\{s \in \Gamma\left(\left(V_{0} \otimes \mathbb{H}\right)^{\mathbb{R}}\right) \mid \mathcal{D} s=0\right\} \text { if } V_{0} \text { has a quaternion structure, } \\
\left\{s \in \Gamma\left(\left(\left(V_{0} \oplus V_{0}^{*}\right) \otimes \mathbb{H}\right)^{\mathbb{R}}\right) \mid \mathcal{D} s=0\right\} \text { otherwise. }
\end{array}\right.
$$

If the real rank of $F$ is denoted by $4 k$, then we can define a mapping $f$ from $G / P$ into a real Grassmannian manifold $G r_{4 k}\left(W^{\mathbb{R}^{*}}\right)$. To do so, note that the evaluation mapping

$$
W^{\mathbb{R}} \rightarrow F
$$

is surjective by BBW, so we obtain an exact sequence of vector bundles:

$$
0 \rightarrow V_{1} \rightarrow \underline{W^{\mathbb{R}}} \rightarrow F \rightarrow 0,
$$

where $\underline{W}^{\mathbb{R}}$ is a trivial vector bundle $G / P \times W^{\mathbb{R}} \rightarrow G / P$. Then, since any fiber $V_{1_{[g]}}$ of $V_{1} \rightarrow G / P$ can be considered as a $4 k$-codimensional subspace of $W^{\mathbb{R}}$, the subspace $V_{1_{[g]}}$ determines a $4 k$-dimensional subspace of $W^{\mathbb{R}^{*}}$. Hence a mapping $f: G / P \rightarrow G r_{4 k}\left(W^{\mathbb{R}^{*}}\right)$ can be defined by

$$
f([g])=V_{1_{[g]}}:=\left\{s \in W^{\mathbb{R}} \mid s[g]=0\right\} .
$$

Theorem 6.1. A mapping $f: G / P \rightarrow G r_{4 k}\left(W^{\mathbb{R}^{*}}\right)$ is an embedding.

Proof. By definition, $f$ is $G$-equivariant and the image of $f$ is a $G$-orbit in $G r_{4 k}\left(W^{\mathbb{R}^{*}}\right)$. We fix $V_{1_{[e]}}$ and denote the isotropy subgroup by $H_{1}$. Then the image of $f$ can be identified with a homogeneous space $G / H_{1}$. Since $P$ acts on $V_{1_{[e]}}$, we have $P \subset H_{1}$. On the other hand, $P$ is a maximal connected subgroup of $G$, because $G / P$ is a symmetric space. Hence $H_{1}$ must be a finite covering of $P$. (It is clear that $f$ is not a constant mapping and so, $H_{1} \neq G$.) In particular, 
$G / H_{1}$ has an induced quaternion-Kähler structure with a positive Ricci curvature. The property of a positive quaternion-Kähler manifold of being simply connected implies that $H_{1}=P$.

Using metrics, we can also define an embedding from $G / P$ into $G r_{4 k}\left(W^{\mathbb{R}}\right)$. This mapping is denoted by the same symbol $f: G / P \rightarrow G r_{4 k}\left(W^{\mathbb{R}}\right)$. We call $f: G / P \rightarrow G r_{4 k}\left(W^{\mathbb{R}}\right)$ the twistor embedding associated with $F \rightarrow G / P$.

A twistor embedding associated with an irreducible homogeneous vector bundle $F \rightarrow G / P$ is $G$-equivariant, and the image of $f$, denoted by $\operatorname{Im} f$, is one orbit in $G r_{4 k}\left(W^{\mathbb{R}}\right)$. Since $W^{\mathbb{R}}$ has a $G$-invariant inner product, a Grassmannian $G r_{4 k}\left(W^{\mathbb{R}}\right)$ has a natural $G$-invariant metric, which makes the Lie group $G$ be an isometry group of $G r_{4 k}\left(W^{\mathbb{R}}\right)$. Hence, if $\operatorname{Im} f$ is a non-principal orbit, then Hsiang-Lawson's theorem [9, Corollary 1.1] can be applied to show that the twistor embedding associated with $F$ is minimal. To know whether $\operatorname{Im} f$ is a principal orbit or not, we may only check the slice representation, which is a $P$-representation to the normal space of $\operatorname{Im} f$ in the tangent space of $G r_{4 k}\left(W^{\mathbb{R}}\right)$ at one point. Indeed, if the slice representation is not trivial, then $\operatorname{Im} f$ is a non-principal orbit. Fortunately, it is easy to obtain the slice representation. First, we divide $W^{\mathbb{R}}$ into $P$-representation spaces:

$$
W=V_{0} \otimes \mathbb{H} \oplus V_{1},
$$

where $V_{1}$ is a representation of $K$ and we omit the symbol ${ }^{\mathbb{R}}$. Then the tangent space of $G r_{4 k}\left(W^{\mathbb{R}}\right)$ is identified with

$$
\left(V_{0} \otimes \mathbb{H}\right) \otimes V_{1} \cong\left(V_{0} \otimes V_{1}\right) \otimes \mathbb{H} .
$$

The tangent space of $G / P$ can be expressed as $V \otimes \mathbb{H}$, where $V$ is an irreducible representation of $K$. Consequently, Schur's lemma yields that the normal space is also expressed as $V_{N} \otimes \mathbb{H}$, where $V_{N}$ is an appropriate representation of $K$ such that $V \oplus V_{N} \cong V_{0} \otimes V_{1}$. Since at least $\operatorname{Sp}(1)$ acts non-trivially on $\mathbb{H}$, the slice representation is not trivial as a representation of $P$.

Theorem 6.2. Let $F$ be an irreducible homogeneous vector bundle identified with $V_{0} \otimes \mathbb{H}$ on the Wolf space $G / P$. Then the twistor embedding $f: G / P \rightarrow G r_{4 k}\left(W^{\mathbb{R}}\right)$ is a minimal embedding.

We list examples of twistor embeddings associated with vector bundles in Theorem 4.1. Choosing an orthogonal basis of $W^{\mathbb{R}}$, we identify $W^{\mathbb{R}}$ with $\mathbb{R}^{N}$.

\section{Example.}

$$
\begin{aligned}
& \text { - } G_{2}\left(\mathbb{C}^{n+2}\right) \rightarrow G r_{4}\left(\mathbb{R}^{2 n+2}\right), \quad \bullet G_{4}\left(\mathbb{R}^{n+4}\right) \rightarrow G r_{4}\left(\mathbb{R}^{n+4}\right), \\
& \text { - } G_{4}\left(\mathbb{R}^{7}\right) \rightarrow G r_{4}\left(\mathbb{R}^{8}\right), \quad \quad \bullet G_{4}\left(\mathbb{R}^{8}\right) \rightarrow G r_{4}\left(\mathbb{R}^{8}\right), \\
& \text { - } G_{4}\left(\mathbb{R}^{9}\right) \rightarrow G r_{8}\left(\mathbb{R}^{16}\right), \quad \bullet G_{4}\left(\mathbb{R}^{10}\right) \rightarrow G r_{16}\left(\mathbb{R}^{32}\right), \\
& \text { - } \mathbb{H} P^{n} \rightarrow G r_{4}\left(\mathbb{R}^{4 n+4}\right) \text {, } \\
& \text { - } E_{6} / \operatorname{Sp}(1) \operatorname{SU}(6) \rightarrow G r_{24}\left(\mathbb{R}^{54}\right), \quad \bullet E_{7} / \operatorname{Sp}(1) \operatorname{Spin}(12) \rightarrow G r_{48}\left(\mathbb{R}^{112}\right) \text {, } \\
& \text { - } F_{4} / \mathrm{Sp}(1) \mathrm{Sp}(3) \rightarrow G r_{12}\left(\mathbb{R}^{26}\right), \quad \bullet G_{2} / \mathrm{SO}(4) \rightarrow G r_{4}\left(\mathbb{R}^{7}\right) \text {. }
\end{aligned}
$$

We list examples of twistor embeddings associated with vector bundles in section 5. Choosing an orthogonal basis of $W^{\mathbb{R}}$, we identify $W^{\mathbb{R}}$ with $\mathbb{R}^{N}$.

\section{Example.}

$$
\begin{aligned}
& \bullet \mathbb{H} P^{n} \rightarrow G r_{4 n}\left(\mathbb{R}^{2 n^{2}+3 n}\right), \\
& \bullet G r_{2}\left(\mathbb{C}^{n+2}\right) \rightarrow G r_{4 n}\left(\mathbb{R}^{n^{2}+3 n+2}\right), \\
& \bullet G r_{4}\left(\mathbb{R}^{12}\right) \rightarrow G r_{32}\left(\mathbb{R}^{64}\right)
\end{aligned}
$$


TABLE 7.1

\begin{tabular}{|c|c|c|c|c|c|c|}
\hline$G$ & $\mathrm{SU}(n)$ & \multicolumn{2}{|c|}{$\operatorname{Spin}(n)$} & $\operatorname{Spin}(7$ & Spin(9) & $\operatorname{Sp}(n)$ \\
\hline$V_{0}$ & $\mathbb{C}, \mathbb{C}^{*}$ & \multicolumn{2}{|c|}{$\mathbb{C}_{\mathbb{H}}^{2}$} & $S_{\mathbb{H}}$ & $S_{\mathbb{H}}$ & $\mathbb{C}$ \\
\hline \multirow{4}{*}{$W^{-2}$} & $\left(\mathbb{C}^{n} \oplus \mathbb{C}^{n^{*}}\right)^{\mathbb{R}}$ & \multicolumn{2}{|c|}{$\mathbb{R}^{n}$} & $S^{\mathbb{R}}$ & $S^{\mathbb{R}}$ & $\left(\mathbb{H}^{n} \oplus \mathbb{H}^{n}\right)^{\mathbb{R}}$ \\
\hline & & $\bar{G}$ & \multicolumn{2}{|c|}{ Spin(8) } & $G_{2}$ & \\
\hline & & $V_{0}$ & \multicolumn{2}{|c|}{$S_{\mathbb{H}}^{+}, S_{\mathbb{H}}^{-}$} & $\mathbb{C}_{\mathbb{H}}^{2}$ & \\
\hline & & $W^{\mathbb{R}}$ & \multicolumn{2}{|c|}{$S^{+^{\mathbb{R}}}, S^{-\mathbb{R}^{\mathbb{R}}}$} & $\varpi_{1}^{\mathbb{R}}$ & \\
\hline
\end{tabular}

\section{MaXimum of the NORM Squared of the TWistor SECTION}

Let $F$ be an irreducible homogeneous vector bundle in Theorem 4.1. In this section, we consider a function $\|s\|^{2}: M:=G / P \rightarrow \mathbb{R}$, where $\|s\|$ is the pointwise norm of a twistor section. We denote by $S_{M}$ the subset of $M$ on which the function $\|s\|^{2}$ attains the maximum value.

Lemma 7.1. Under the decompositions $W^{\mathbb{R}}=V_{0} \otimes \mathbb{H} \oplus V_{1}$ and $\mathfrak{g}=\mathfrak{p} \oplus \mathfrak{m}, \mathfrak{m}$ acts off-diagonally on $W^{\mathbb{R}}$; in other words, $\mathfrak{m}\left(V_{0} \otimes \mathbb{H}\right) \subset V_{1}$ and $\mathfrak{m} V_{1} \subset V_{0} \otimes \mathbb{H}$.

Proof. As a representation of $\mathfrak{p}=\mathfrak{k} \oplus \mathfrak{s p}(1), \mathfrak{m}$ is expressed as

$$
\mathfrak{m}=V \otimes \mathbb{H} .
$$

The Clebsch-Gordan formula yields that $(V \otimes \mathbb{H}) \otimes\left(V_{0} \otimes \mathbb{H}\right)$ cannot have $V_{0} \otimes \mathbb{H}$ as an irreducible component; thus for an arbitrary $X \in \mathfrak{m}$ and $v_{0} \otimes h \in V_{0} \otimes \mathbb{H}$,

$$
X v_{0} \otimes h \in V_{1} .
$$

In a similar manner, we have for an arbitrary $v_{1} \in V_{1}$,

$$
X v_{1} \in V_{0} \otimes \mathbb{H}
$$

because $(V \otimes \mathbb{H}) \otimes V_{1}$ cannot have $V_{1}$ as an irreducible component.

From now on, we restrict our attention to the case that a principal $G$-orbit in $W^{\mathbb{R}}$ is a maximal dimensional sphere, and such a representation is called a representation of spherical type. From Table 4.3, all bundles $F \cong V_{0} \otimes \mathbb{H}$ which have representations $W^{\mathbb{R}}$ of spherical types arising from real twistor sections can be classified as in Table 7.1.

Our restriction to representations of spherical types, combined with Theorem 4.2 , yields that any $w \in W^{\mathbb{R}} \backslash\{0\}$ corresponds to a transversal twistor section of $F$. To describe a corresponding section $s$ to $w \in W^{\mathbb{R}}$, let $\pi: W^{\mathbb{R}} \rightarrow V_{0} \otimes \mathbb{H}$ be the orthogonal projection and $\pi^{\perp}: W^{\mathbb{R}} \rightarrow V_{1}$ be the complementary orthogonal projection. Then we have

$$
s([g])=\left[g, \pi\left(g^{-1} w\right)\right] .
$$

We fix the notation $w \in W^{\mathbb{R}} \backslash\{0\}$ and the twistor section $s \in \Gamma(F)$ corresponding to $w$ throughout this section.

Lemma 7.2. The covariant derivative of a twisotr section $s$ is expressed as

$$
\nabla_{X^{M}} s=\left[g,-\pi_{\mathfrak{m}}\left(\operatorname{Ad}\left(g^{-1}\right) X\right) \pi^{\perp}\left(g^{-1} w\right)\right],
$$

where $X \in \mathfrak{g}$ and $X^{M}$ is the Killing vector field on $M$ generated by $X$. 
Proof. If the twistor section $s$ is identified with the corresponding function $\pi\left(g^{-1} w\right)$ on $G$ with values in $V_{0} \otimes \mathbb{H}$, then we have

$$
\begin{aligned}
& -\pi\left(\operatorname{Ad}\left(g^{-1}\right) X g^{-1} w\right)+\pi_{\mathfrak{k}}\left(\operatorname{Ad}\left(g^{-1}\right) X\right) \pi\left(g^{-1} w\right) \\
=- & \pi_{\mathfrak{k}}\left(\operatorname{Ad}\left(g^{-1}\right) X\right) \pi\left(g^{-1} w\right)-\pi_{\mathfrak{m}}\left(\operatorname{Ad}\left(g^{-1}\right) X\right) \pi^{\perp}\left(g^{-1} w\right) \\
& \left.\quad+\pi_{\mathfrak{k}}\left(\operatorname{Ad}\left(g^{-1}\right) X\right)\right) \pi\left(g^{-1} w\right) \\
= & -\pi_{\mathfrak{m}}\left(\operatorname{Ad}\left(g^{-1}\right) X\right) \pi^{\perp}\left(g^{-1} w\right) .
\end{aligned}
$$

Here we use Lemma 7.1 to obtain

$$
\begin{aligned}
& \pi\left(\operatorname{Ad}\left(g^{-1}\right) X g^{-1} w\right) \\
& =\pi\left(\pi_{\mathfrak{k}}\left(\operatorname{Ad}\left(g^{-1}\right) X\right) g^{-1} w\right)+\pi\left(\pi_{\mathfrak{m}}\left(\operatorname{Ad}\left(g^{-1}\right) X\right) g^{-1} w\right) \\
& =\pi_{\mathfrak{k}}\left(\operatorname{Ad}\left(g^{-1}\right) X\right) \pi\left(g^{-1} w\right)+\pi_{\mathfrak{m}}\left(\operatorname{Ad}\left(g^{-1}\right) X\right) \pi^{\perp}\left(g^{-1} w\right),
\end{aligned}
$$

as required.

Using the covariant derivative of $s$, we can obtain the critical sets of the function $\|s\|^{2}: M:=G / P \rightarrow \mathbb{R}$.

Lemma 7.3. The critical sets of the function $\|s\|^{2}$ consists of $S$ and $S_{M}$.

Proof. It follows from Lemma 7.2 that

$$
\begin{aligned}
d\|s\|^{2}\left(X^{M}\right) & =2\left\langle\nabla_{X^{M}} s, s\right\rangle \\
& =-2\left\langle\pi_{\mathfrak{m}}\left(\operatorname{Ad}\left(g^{-1}\right) X\right) \pi^{\perp}\left(g^{-1} w\right), \pi\left(g^{-1} w\right)\right\rangle .
\end{aligned}
$$

Lemma 7.1 yields that

$$
d\|s\|^{2}\left(X^{M}\right)=-2\left\langle\operatorname{Ad}\left(g^{-1}\right) X \pi^{\perp}\left(g^{-1} w\right), \pi\left(g^{-1} w\right)\right\rangle .
$$

Now we suppose that $[g] \in G / P$ is a critical point of the function $\|s\|^{2}$; in other words, $d\|s\|^{2}\left(X^{M}\right)=0$ for all $X \in \mathfrak{g}$. Since a representation $W^{\mathbb{R}}$ is of spherical type, the subspace of $W^{\mathbb{R}}$ spanned by vectors of the form $\operatorname{Ad}\left(g^{-1}\right) X \pi^{\perp}\left(g^{-1} w\right)(X \in \mathfrak{g})$ can be identified with the tangent space of a sphere $G \pi^{\perp}\left(g^{-1} w\right) \subset W^{\mathbb{R}}$ at a point $\pi^{\perp}\left(g^{-1} w\right)$ if $\pi^{\perp}\left(g^{-1} w\right) \neq 0$. It follows that $\pi\left(g^{-1} w\right)$ is parallel to $\pi^{\perp}\left(g^{-1} w\right)$, so we have

$$
\pi\left(g^{-1} w\right)=0, \quad \text { in a similar manner, or } \quad \pi^{\perp}\left(g^{-1} w\right)=0 .
$$

When $\pi\left(g^{-1} w\right)=0$, we get $[g] \in S$. If $\pi^{\perp}\left(g^{-1} w\right)=0$, then $\pi\left(g^{-1} w\right)=g^{-1} w$. The inequality $\|s\|^{2}([g])=\left\|\pi\left(g^{-1} w\right)\right\|^{2} \leqq\left\|g^{-1} w\right\|^{2}=\|w\|^{2}$ gives $[g] \in S_{M}$.

Remark. We also know that

$$
S_{M}=\left\{[g] \in G / P \mid \pi^{\perp}\left(g^{-1} w\right)=0\right\} .
$$

Lemma 7.4. The covariant derivative $\nabla s$ vanishes on $S_{M}$.

Proof. Lemma 7.2 and (3) yield that $\nabla s=0$ on $S_{M}$.

Definition 7.5. For the same $w \in W^{\mathbb{R}}$ as in the definition of the twistor section $s([g])=\left[g, \pi\left(g^{-1} w\right)\right]$ of $F \cong V_{0} \otimes \mathbb{H}$, we define a section $s^{\perp}$ of $V_{1}$ as

$$
s^{\perp}([g])=\left[g, \pi^{\perp}\left(g^{-1} w\right)\right] \in \Gamma\left(V_{1}\right) .
$$


Since $W^{\mathbb{R}}$ is a representation of spherical type, the zero locus $S_{0}^{\perp}$ of $s^{\perp}$ is not an empty set. Then, Sard's theorem yields that generic sections are transverse to the zero section and our assumption for $W^{\mathbb{R}}$ implies that $s^{\perp}$ is also a transversal section of $V_{1}$.

Lemma 7.6. In our notation, we have

$$
S_{M}=S_{0}^{\perp} .
$$

In particular, the subset $S_{M}$ is a submanifold of $M$ and the dimension of $S_{M}$ is equal to $\operatorname{dim} M-\operatorname{rank} V_{1}$.

Proof. $S_{0}^{\perp}$ can be written as

$$
S_{0}^{\perp}=\left\{[g] \in G / P \mid \pi^{\perp}\left(g^{-1} w\right)=0\right\},
$$

which is the same set as $S_{M}$. The transversality of $s^{\perp}$ yields the result.

The tangent space of $S_{M}$ is expressed as

$$
\begin{aligned}
T S_{M_{[g]}} & =\left\{X^{M} \in T M_{[g]} \mid \nabla_{X^{M}}^{1} s^{\perp}=0\right\} \\
& =\left\{X^{M} \in T M_{[g]} \mid \pi_{\mathfrak{m}}\left(\operatorname{Ad}\left(g^{-1}\right) X\right) \pi\left(g^{-1} w\right)=0\right\},
\end{aligned}
$$

where $\nabla^{1}$ is the canonical connection of $V_{1}$.

Obviously, we have

Lemma 7.7. Let $H$ be the isotropy subgroup of $G$ at $w \in W^{\mathbb{R}}$ as in Theorem 4.2. Then $H$ acts on $S_{M}$.

Using again Hsiang-Hsiang's result [8], we can know the isotropy subgroup $H$. In particular, $H$ is connected. This implies the connectivity of $S_{M}$.

Lemma 7.8. The submanifold $S_{M}$ is connected. Moreover, $H$ acts on $S_{M}$ transitively.

Proof. We do not have any convenient criterion such as that in Proposition 3.9 for a similar result on $S$. However, case by case, we can check that $V_{0} \otimes \mathbb{H}$ is a representation of spherical type, as a representation of $P$. Hence, if $\left[g_{1}\right]$ and $\left[g_{2}\right]$ are in $S_{M}$, in other words, $g_{1}^{-1} w, g_{2}^{-1} w \in V_{0} \otimes \mathbb{H}$, then we can find an element $k \in P$ such that

$$
k g_{1}^{-1} w=g_{2}^{-1} w \Longleftrightarrow g_{2} k g_{1}^{-1} \in H .
$$

This means that a point $\left[g_{2}\right] \in M$ is in the $H$-orbit $H\left[g_{1}\right] \subset M$.

From Lemma 7.8, we know that $S_{M}$ is also an $H$-orbit in $M$. Consequently, $S_{M}$ can be expressed as $H / H \cap P$, and $H \cap P$ can be obtained as the isotropy subgroup of $P$ at a point in $V_{0} \otimes \mathbb{H} \backslash\{0\}$. In this case, $\operatorname{Sp}(1) \subset P$ acts non-trivially on $V_{0} \otimes \mathbb{H}$. Since $\operatorname{Sp}(1) \subset P$ acts trivially on $V_{1}$, the zero locus $S$ is a quaternion submanifold. In this sense, $S_{M}$ is conceptually far from a quaternion submanifold. (In particular, Hsiang-Lawson's result [9, Corollary 1.1] also yields that $S_{M}$ is a minimal submanifold, because the normal bundle of $S_{M}$ can be identified with $V_{1}$ (Lemma 7.6 and transversality) and so, the slice representation is not trivial. We can now list $S_{M}$ in each Wolf space.

Theorem 7.9. The subsets $S_{M}$ are classified in Table 7.2. One consequence is that $S_{M}$ is a totally geodesic submanifold of $M$. 
TABLE 7.2

\begin{tabular}{|c|c|c|c|c|c|c|}
\hline$G$ & $\overline{\mathrm{SU}}(n)$ & $\operatorname{Spin}(n)$ & \multicolumn{2}{|c|}{ Spin(7) } & Spin(9) & $\operatorname{Sp}(n)$ \\
\hline$\overline{S_{M}}$ & $\mathbb{C} P^{n-2}$ & $G r_{3}\left(\mathbb{R}^{n-1}\right)$ & \multicolumn{2}{|c|}{$G_{2} / S O(4)$} & $G r_{4}\left(\mathbb{R}^{7}\right)$ & $1 p t$ \\
\hline & $\bar{G}$ & \multicolumn{2}{|c|}{ Spin(8) } & \multicolumn{2}{|r|}{$\overline{\overline{G_{2}}}$} & \\
\hline & $\overline{S_{M}}$ & \multicolumn{2}{|c|}{$G r_{4}\left(\mathbb{R}^{7}\right), G r_{4}\left(\mathbb{R}^{7}\right)$} & \multicolumn{2}{|c|}{$\mathrm{SU}(3) / \mathrm{SO}(3)$} & \\
\hline
\end{tabular}

Finally, we are interested in relating our theory to the topology of Wolf spaces. Using the function $\|s\|^{2}$, for instance, we can know the topology of $M$ from those of $S$ and $S_{M}$ up to homotopy.

Lemma 7.10. Under the hypothesis that $W^{\mathbb{R}}$ is a representation of spherical type, $\|s\|^{2}$ is a Morse function in the sense of Bott.

Proof. First of all, we compute $\nabla^{2} s$ on $S_{M}$. From Lemma 7.4 we have

$$
\nabla^{2} s\left(X^{M}, Y^{M}\right)=\nabla_{X^{M}}\left(\nabla_{Y^{M}} s\right)-\nabla_{\nabla_{X^{M}} Y^{M}} s=\nabla_{X^{M}}\left(\nabla_{Y^{M}} s\right),
$$

for an arbitrary $X, Y \in \mathfrak{g}$ on $S_{M}$. Then a direct computation implies that

$$
\nabla_{X^{M}}\left(\nabla_{Y^{M}} s\right)=\left[g, \pi_{\mathfrak{m}}\left(\operatorname{Ad}\left(g^{-1}\right) Y\right) \pi_{\mathfrak{m}}\left(\operatorname{Ad}\left(g^{-1}\right) X\right) \pi\left(g^{-1} w\right)\right],
$$

and we obatin

$$
\nabla^{2} s\left(X^{M}, Y^{M}\right)=\left[g, \pi_{\mathfrak{m}}\left(\operatorname{Ad}\left(g^{-1}\right) Y\right) \pi_{\mathfrak{m}}\left(\operatorname{Ad}\left(g^{-1}\right) X\right) \pi\left(g^{-1} w\right)\right] .
$$

Since $\nabla s=0$ on $S_{M}$ and $\nabla d\|s\|^{2}=2\left\{\left\langle\nabla^{2} s, s\right\rangle+\langle\nabla s, \nabla s\rangle\right\}$, we get

$$
\begin{aligned}
\nabla d\|s\|^{2} & \left(X^{M}, Y^{M}\right) \\
& =2\left\langle\pi_{\mathfrak{m}}\left(\operatorname{Ad}\left(g^{-1}\right) Y\right) \pi_{\mathfrak{m}}\left(\operatorname{Ad}\left(g^{-1}\right) X\right) \pi\left(g^{-1} w\right), \pi\left(g^{-1} w\right)\right\rangle_{V_{0} \otimes \mathbb{H}} \\
& =-2\left\langle\pi_{\mathfrak{m}}\left(\operatorname{Ad}\left(g^{-1}\right) X\right) \pi\left(g^{-1} w\right), \pi_{\mathfrak{m}}\left(\operatorname{Ad}\left(g^{-1}\right) Y\right) \pi\left(g^{-1} w\right)\right\rangle_{W^{\mathbb{R}}}
\end{aligned}
$$

on $S_{M}$. In particular, we have

$$
\begin{aligned}
& \nabla d\|s\|^{2}\left(X^{M}, X^{M}\right) \\
= & -2\left\langle\pi_{\mathfrak{m}}\left(\operatorname{Ad}\left(g^{-1}\right) X\right) \pi\left(g^{-1} w\right), \pi_{\mathfrak{m}}\left(\operatorname{Ad}\left(g^{-1}\right) X\right) \pi\left(g^{-1} w\right)\right\rangle_{W^{\mathbb{R}}}
\end{aligned}
$$

on $S_{M}$. Hence

$$
\nabla d\|s\|^{2}\left(X^{M}, X^{M}\right)=0 \Longleftrightarrow \pi_{\mathfrak{m}}\left(\operatorname{Ad}\left(g^{-1}\right) X\right) \pi\left(g^{-1} w\right)=0 .
$$

The latter condition is equivalent to $X^{M} \in T S_{M}$, because

$$
T S_{M_{[g]}}=\left\{X^{M} \in T M_{[g]} \mid \pi_{\mathfrak{m}}\left(\operatorname{Ad}\left(g^{-1}\right) X\right) \pi\left(g^{-1} w\right)=0\right\} .
$$

On the zero locus $S$, we have $\nabla d\|s\|^{2}=2\langle\nabla s, \nabla s\rangle$. It follows from Lemma 7.2 that

$$
\begin{aligned}
& \nabla d\|s\|^{2}\left(X^{M}, X^{M}\right) \\
& =2\left\langle\pi_{\mathfrak{m}}\left(\operatorname{Ad}\left(g^{-1}\right) X\right) \pi^{\perp}\left(g^{-1} w\right), \pi_{\mathfrak{m}}\left(\operatorname{Ad}\left(g^{-1}\right) X\right) \pi^{\perp}\left(g^{-1} w\right)\right\rangle_{W_{\mathbb{R}}^{\mathbb{R}}}
\end{aligned}
$$

Hence

$$
\nabla d\|s\|^{2}\left(X^{M}, X^{M}\right)=0 \Longleftrightarrow \pi_{\mathfrak{m}}\left(\operatorname{Ad}\left(g^{-1}\right) X\right) \pi^{\perp}\left(g^{-1} w\right)=0 .
$$

The latter condition is equivalent to $X^{M} \in T S_{0}$, because, as we have already seen,

$$
S=\left\{[g] \in G / P \mid \pi\left(g^{-1} w\right)=0\right\},
$$


and the tangent space of $S$ is expressed as

$$
\begin{aligned}
T S_{[g]} & =\left\{X^{M} \in T M_{[g]} \mid \nabla_{X^{M}} s=0\right\} \\
& =\left\{X^{M} \in T M_{[g]} \mid \pi_{\mathfrak{m}}\left(\operatorname{Ad}\left(g^{-1}\right) X\right) \pi^{\perp}\left(g^{-1} w\right)=0\right\},
\end{aligned}
$$

as required.

\section{REFERENCES}

[1] M.F. Atiyah, N.J. Hitchin and I.M. Singer, Self-duality in four-dimensional Riemannian geometry, Proc. Roy. Soc. London 362 (1978), 425-461. MR506229 (80d:53023)

[2] E. Bonan, Tenseur de structure d'une variété presque quaternioniennes, C. R. Acad. Sci. Paris, 259 (1964), 45-48. MR0166741 (29:4014)

[3] R. Bott, Homogeneous vector bundles, Ann. of Math. 66 (1957), 203-248. MR0089473 (19:681d)

[4] N. Bourbaki, "Groupes et algèbres de Lie", Hermann, Paris (1975). MR0453824 (56:12077)

[5] K. Galicki and Y.S. Poon, Duality and Yang-Mills fields on quaternionic Kähler manifolds, J. Math. Phys. 32 (1991), 1263-1268. MR.1103479 (92i:53024)

[6] A. Gray, A note on manifolds whose holonomy group is a subgroup of $\operatorname{Sp}(n) \cdot \operatorname{Sp}(1)$, Michigan Math. J. 16 (1969), 125-128. MR.0244913 (39:6226)

[7] N.J. Hitchin, Kählerian twistor spaces, Proc. London. Math. Soc. (3) 43 (1981), 133-150. MR623721 (84b:32014)

[8] W.C. Hsiang and W.Y. Hsiang, Differential actions of compact connected classical groups: II, Ann. of Math. 92 (1970), 189-223. MR0265511 (42:420)

[9] W.Y. Hsiang and H.B. Lawson, Minimal Submanifolds of Low Cohomogeneity, J. Differential Geometry. 5 (1971), 1-38. MR0298593 (45:7645)

[10] B. Kostant, Lie algebra cohomology and the generalized Borel-Weil Theorem, Ann. of Math. 74 (1961), 329-387. MR0142696 (26:265)

[11] C. LeBrun, Fano Manifolds, Contact Structures, and Quaternionic Geometry, International J. Math. 6 (1995), 419-437. MR1327157 (96c:53108)

[12] C. LeBrun and S.M. Salamon, Strong rigidity of positive quaternion-Kähler manifolds, Invent. Math. 118 (1994), 109-132. MR 1288469 (95k:53059)

[13] M. Mamone Capria and S.M. Salamon, Yang-Mills fields on quaternionic spaces, Nonlinearity 1 (1988), 517-530. MR967469 (89k:58064)

[14] Y. Nagatomo, Examples of vector bundles admitting unique ASD connections on quaternionKähler manifolds, Proc. Amer. Math. Soc. 127 (1999), 3043-3048. MR1616637(2000a:53042)

[15] Y. Nagatomo, Representation theory and ADHM-construction on quaternion symmetric spaces, Trans. Amer. Math. Soc. 353 (2001), 4333-4355. MR.1851173 (2002m:53075)

[16] Y. Nagatomo, Geometry of the Twistor Equation and its Applications, Contemporary Mathematics 309 (2002), 165-176. MR.1953358 (2003k:53055)

[17] Y. Nagatomo and T. Nitta, Vanishing theorem for quaternionic complexes, Bull. London Math. Soc. 29 (1997), 359-366. MR1435574 (98b:32028)

[18] S.M. Salamon, Quaternionic Kähler Manifolds, Invent. Math. 67 (1982), 143-171. MR664330 (83k:53054)

[19] S.M. Salamon, Differential geometry of quaternionic manifolds, Ann. Sci. Ecole Norm. Sup. 19 (1986), 31-55. MR860810 (87m:53079)

[20] H. Tasaki, Quaternionic Submanifolds in Quaternionic Symmetric Spaces, Tôhoku Math. J. 38 (1986), 513-538. MR 867059 (87k:53124)

[21] G. Tian, Gauge Theory and calibrated geometry, I, Ann. of Math. 151 (2000), 193-268. MR.1745014 (2000m:53074)

[22] M. Verbitsky, Hyperholomorphic bundles over a hyper-Kähler manifold, J. Alg. Geom. 5 (1996), 633-669. MR.1486984 (2000a:32051)

[23] J.A. Wolf, Complex homogeneous contact manifolds and quaternionic symmetric spaces, J. Math. Mech. 14 (1965), 1033-1047. MR0185554 (32:3020)

Graduate School of Mathematics, Kyushu University, Ropponmatsu, Fukuoka 8108560, JAPAN

E-mail address: nagatomo@math.kyushu-u.ac.jp 